\title{
The Second-Order Sharpening of Blurred Smooth Borders*
}

\author{
By Blair Swartz \\ In appreciation, to Eugene and Muriel Isaacson
}

\begin{abstract}
The problem is to approximate, with local second-order accuracy, the smooth boundary separating a black and a white region in the plane, given discretely located gray values associated with a blurring of that border. "Second-order", here, is with respect to the size $h$ of the scale of the prescribed blurring. The locally determined approximations are line segments. The algorithms discussed here can result in secondorder accuracy, but they may not in certain geometric circumstances. Typical local curvature estimates based on adjacent line segments do not converge, but an atypical one does. Consideration of a class of scaled blurrings leads to a type of blurring of borders which is particularly easy for a computer to undo locally, yielding a line which is locally second-order accurate. Some extensions to three (and more) dimensions are appended.
\end{abstract}

1. Introduction, Color Functions, One Dimension, Summary. This work was motivated initially by the following facts: In computational fluid dynamics various regions of the fluid have their own physically important characteristics. The volume-of-fluid method (e.g., Nichols and Hirt [22]) keeps track of interfaces between such regions by assuming each is colored its own uniform, physically irrelevant, color; and a transport equation is added to move these colors. With this, local movement of an interface takes place at the local fluid velocity. In the discretization of the color's transport equation, the numerical quantities kept track of are the average color of each of the (congruent) square cells of the computational mesh. The SLIC algorithm (Noh and Woodward [23]) uses similar quantities to locate interfaces. For recent work concerning interface approximation in computational hydrodynamics, see Hyman [19, p.402].

This paper provides a framework, with an initial analysis, for the approximate reconstruction of borders using discretely located values of locally averaged colors; and it proposes certain algorithms possessing the potential for second-order accuracy-second-order, that is, in terms of the size of the scale of the prescribed blurring. This potential proves realizable for many types of scaled blurring and geometric circumstances - but not necessarily for all. Lack of second-order accuracy for an algorithm reproducing linear interfaces is of particular interest to the numerical

Received September 21, 1987; revised September 6, 1988.

1980 Mathematics Subject Classification (1985 Revision). Primary 65D10; Secondary 65D05, 65M99, 65N99, 41 A05, 41A63.

Key words and phrases. Blurred, boundary, border, edge, curve, surface, interface, approximation, reconstruction.

*Supported by the U. S. Department of Energy under contracts KC-07-01-01-0 and W-7405ENG.36. The publisher recognizes the U. S. Government retains a nonexclusive, royalty-free license to publish or reproduce the published form of this contribution, or to allow others to do so, for U. S. Government purposes. 
analyst, as is the complete lack of convergence of the simplest curvature-estimating algorithms based on the rate that approximate tangents seem to turn. Only brief comment is made concerning applications to computational hydrodynamics.

Let us idealize the problem as follows. The plane is decomposed into two regions, $\Omega_{0}$ and $\Omega_{1}$, separated by their common border (boundary, edge, interface) $\beta$-this bordering curve is assumed to have bounded curvature, to not cross itself, and to consist of one component only. Associate with this setup the color function, $c$, defined by

$$
c(x):=\left\{\begin{array}{ll}
0, & x \text { in } \Omega_{0}, \\
1, & x \text { in } \Omega_{1}, \\
1 / 2, & x \text { in } \beta .
\end{array}\right. \text { and }
$$

In particular, and somewhat artificially, $\beta$ is the inverse image of $1 / 2$ under the map $c$. Let $S(x, h)$ be the square of area $h^{2}$, centered on the point $x$, and with sides parallel to the coordinate axes. Associate with $c$ and $S$ the "average color" function

$$
\bar{c}(x):=\iint_{S(x, h)} c(\xi) d A(\xi) / h^{2}, \quad x \text { in the plane. }
$$

For $h$ sufficiently small (relative to $\beta$ 's curvature), the set $\bar{c}^{-1}(1 / 2)$ is 1 -dimensional and approximates $\beta$. Given a uniform mesh of points $X_{i}:=\left(i_{1} h, i_{2} h\right)$ of gridsize $h$-the same $h$ as in $S$-we shall consider the use of meshpoint values $\bar{c}_{i}:=\bar{c}\left(X_{i}\right)$ in approximating $\beta$.

Indeed, such local averaging makes it possible to reconstruct a piecewise constant function of one variable, and the associated algorithm is completely understood. In this context, there is clearly not enough information in the values $c\left(x_{i}\right)$ of the function $c(x):=0, x<d ; c(x):=1$, otherwise, on a uniform mesh $x_{i}:=i h$, to allow one to locate the discontinuity better than finding the two mesh points between which it lies. Yet, a single value $\bar{c}\left(x_{j}\right)$, of the corresponding average color function $\bar{c}(x)$ (i.e., $c$ averaged over an interval of length $h$ centered on $x$ ), enables one to locate $d$ perfectly, if that value is the one meshpoint value of $\bar{c}$ which lies strictly between zero and one--use the known behavior of $\bar{c}$ nearby (namely, linear, with slope $1 / h$ ) to locate $d$ relative to $x_{j}$ via $\bar{c}(d)=1 / 2$. (If there is no such value, then $d$ lies precisely halfway between the two meshpoints on which $\bar{c}_{i}$ changes). Moreover, second-order, i.e., $O\left(h^{2}\right)$, accuracy in the location of $d$ is feasible as long as (a) perturbations $x_{j} \rightarrow \tilde{x}_{j}$ and $\bar{c}\left(x_{j}\right) \rightarrow \tilde{c}_{j}$ are second- and first-order, respectively, while (b) $\tilde{c}_{j}$ still lies strictly between the left and right limits---in particular, were the jump in $c$ of order one, its left and right limits known within $O(h)$, and $d c / d x$ bounded on a punctured neighborhood centered on $d$ and of length at least $2 h$. To complete this one-dimensional example, we note that the similar use of other local linear approximants (e.g., the interpolant of both the gray value and the nearest black/white value which, with it, embrace 1/2) yield an $O(h)$ (but generally not $O\left(h^{2}\right)$ ) accurate estimate of $d$.

This blurring of a discontinuity and the subsequent reconstruction of its location exemplifies one technique for representing a "sub-grid scale" quantity on a grid in such a way that it can be accurately recovered. 
We shall explore the extent that these ideal results extend to two-eventually, more-dimensions. As above, our approach will be based more on the blurring than on its rates of change-more on pinpointing the peal than the ha-ha (to make a metaphor, in view of their possible cross-sections (Huxley [18, p. 97]), of those border-delimiting constructs of 18 th century landscape architecture). The exposition begins by developing notions necessary for border reconstruction using the example of values of the particular average coloring (1.2) on a uniform $h \times h$ mesh. In this connection, Section 2 observes that first-order $(O(h))$ accurate border approximation is relatively easy, but that the use of level curves of certain local polynomial interpolants will not improve this order of accuracy. Section 3 suggests that algorithms which reproduce arbitrary linear borders could lead to higher-order accuracy for curved borders, it defines notions appropriate to such reconstruction (such as the behavior of the average color as one continuously crosses a linear border perpendicularly) and exemplifies them with the orientation-dependent quadratic splines (3.2) associated with the average coloring (1.2) ( $n$th degree splines for polyhedra in $n$ dimensions - see Appendix 4); it then completes proof of the mere first-order accuracy of the appropriate level line of the linear interpolant of three gray colors (1.2) (cf. Counterexample 3.1), and it mentions the previous work in computational hydrodynamics (by DeBar and, later, Youngs) which has utilized the exact reconstruction of linear borders from meshpoint values of (1.2).

The dependence on orientation of the behavior of the average color (1.2) as one crosses a linear border perpendicularly complicates the reconstruction of such borders. A more general moving average is defined in Section 4-it utilizes a scale $h$ which localizes its effect as $h$ gets small, and it contains (1.2) as a special case. It also contains other local averages whose perpendicular border-crossing behavior is independent of orientation, a circumstance allowing particularly simple reconstruction of linear borders from two exact and discretely located gray values. (As with the sign of a square root, there are generally two linear borders which solve the problem-i.e., yield the two gray values - and additional data about the context (like a third value of the average color) is necessary to choose between them). Cf. Sections 5-7 and Summary 7.1. The associated Algorithm 7.1 depends nonlinearly on its data, and it need not yield a line if it is given incorrect data. Moreover, its stability (linearized) can depend on orientation (Proposition 8.1). Hence, as the curvature of a curved border perturbs the gray values one would associate with a local tangent half-space (by order $h$ as $h$ gets small--Remark 8.1), the accuracy of the line the algorithm produces from these perturbed values, although usually of order $h^{2}$, can be at least as bad as order $h^{3 / 2}$ in certain geometric circumstances (Propositions 8.2 and 9.1). (However, a footnote points out that, in the application of DeBar's algorithm to curved borders using the gray colors of two $h \times h$ squares having a common side, the accuracy is $O\left(h^{2}\right)$.)

As one moves along near a blurred border, each successive pair of gray colors can now determine its own approximating line segment, and the turning of these segments should allow an estimate of the border's curvature. But the simplest approaches to this prove to be so inaccurate as to not even converge as $h$ gets small and it is only through a careful analysis of the error in the segments' directions (Proposition 9.1) that one can utilize successive pairs of directions to obtain 
a more complex-but now convergent-curvature estimate (Proposition 9.2). In Section 10 we attempt to do for curvature estimation what we succeeded in doing in Section 6 for border approximation; namely, to create a special scaled averaging whose discrete values could allow particularly easy implementation of the algorithm. Unfortunately, the associated integral equation in this instance is not known to have a solution.

Our analysis shows that the quantities entering error estimates, as the scale $h$ of the local average gets small, reflect the dual nature of the data-i.e., of the locally averaged color. From the viewpoint of the border, the local averaging shrinks to a point. Hence, the important characteristics of the border are its characteristics relative to that point-like the border's local curvature. But from the viewpoint of the probability distribution which does the blurring, the border straightens to become a line. Hence, the important characteristics of the probability distribution are its characteristics relative to that line-like the line's various moments, under the induced mass distribution, about the point nearest the origin-moments normalized by the induced mass of the line. Moreover-because we are basically using two local averages to determine a local tangent's direction - it is not surprising that it is actually a difference quotient of these "specific moments" that enters the error estimates.

Since two discretely located gray values of the average color suffice (essentially) to determine a linear border which would yield them (when such a border exists), additional gray values are redundant for that purpose. Yet, some algorithms already extant use more than two values. For example, Youngs' algorithm uses all nine average colors in a $3 \times 3$ pile of mesh squares. In Sections 11 and 12, we present two algorithms; each is based on three gray values. Section 11's algorithm was designed to avoid iteration-even when the behavior of the local average upon perpendicularly crossing a linear border depends upon orientation. The algorithm is novel in that it requires two gray values to be located far enough from a third, midway point, to allow an explicit and convergent estimate of the border's direction nearby. The line produced by this algorithm is proved second-order accurate near the midway point - in spite of the fact that it fails to reproduce even linear borders. Section 12's algorithm involves data from three neighboring but strictly noncolinear locations. It is shown stable (so, second-order accurate since it reproduces linear borders) independent of orientation - at least, for local averages whose behavior when perpendicularly crossing a linear border is independent of orientation.

Some matters associated with the application to computational hydrodynamics, of border reconstruction from the values of scaled blurrings, are brought up briefly in Section 13.

The application of an integral equation to the analysis of unexpectedly accurate edge perception (at the end of Section 6) is our only explicit indication of relevance of this paper to visual matters. However, a referee has kindly noted other points of contact with image processing and computer vision by mentioning the following concepts from those fields: "... approximating lines in pixel grids; the notions of subpixel accuracy in edge detection and of Gaussian convolution for blurring and interpolation. The use of a continuum of scales of blurrings of an image, where the blurring kernel is a Gaussian, is called 'scale-space' in the computer vision 
literature, and level-crossings in scale-space is an active area of interest...". The IEEE's special image processing issue (Hunt [17]) is a standard reference on that general subject.

Although image processing and other visual applications would seem to be associated with scaled blurrings in two dimensions only, computational hydrodynamics is becoming more capable of-and, so, more concerned with-the approximation of interfaces between three-dimensional regions. Appendix 1, then, concerns the extension of many of this paper's two-dimensional notions to $n$ dimensions. Among other things, algorithms are described which determine-without iteration-the (up to two) hyperplanes (if any) which are at $n$ prescribed (signed) distances from $n$ points in general position in $n$-space. Another generalization-namely, the possibility of second-order accurate reconstruction using scaled blurrings which themselves vary nonsmoothly from data-point to data-point (e.g., the average color of triangles in an irregular triangularization of the plane)-is also briefly considered. Finally, another appendix considers the problem of the existence of linear borders which would yield prescribed average colors at specified locations-the reader will recognize this to be a nontrivial generalization of the "ham-sandwich problem" of Steinhaus.

2. First-Order Approximation. It is easy (in principle) to approximate the border $\beta$ to first-order accuracy using the values of $\bar{c}(1.2)$ on a uniform $h \times h$ grid $\left\{X_{i}\right\}$. For, let $B$ be the set of $X_{i}$ on which $\bar{c}$ is strictly between 0 and 1 . Then, because the (Hausdorff) distance of $B$ from $\beta$ is $O(h)$, any curve within $O(h)$ of $B$ is within $O(h)$ of $\beta$ in this sense. (On the other hand, practical algorithms which compute plane curves approximating discrete point sets can be complicated, and they will not be explored in this paper.) A label associated with the significant values of $\bar{c}$ will be useful:

Definition 2.1. The data $\left(X_{i}, \bar{c}_{i}\right), X_{i}$ in $B$, is defined to be the set of "usable data"; similarly, a meshpoint $X_{i}$, or a value $\bar{c}_{i}$ of $\bar{c}$ there, is "usable" if and only if $\bar{c}_{i}$ lies strictly between zero and one.

The average color $\bar{c}(x)(1.2)$ is piecewise smooth; but, as $h$ gets small, the gradient of $\bar{c}$ gets large near the border $\beta$. So it is not surprising that algorithms based on a local linear interpolant, $l$, or a local bilinear interpolant, $b$, of its mesh-point values $\bar{c}_{i}$ can fail to yield second-order accuracy. ( $b$ has a second interpretation: let the piecewise constant coloring $g(x)$ take on, for each $i$, the value $\bar{c}_{i}$ for $x$ in the open $h \times h$ square centered on $X_{i}$. Then, $b$ is the average color $\bar{g}$ of $g$.) The continua $C_{b}:=b^{-1}(1 / 2)$ and $C_{l}:=l^{-1}(1 / 2)$ can be second-order approximations to $\beta$ in special cases: for example, $C_{b}$ and $C_{l}$ actually coincide with $\beta$ when $\beta$ is a straight line which passes through immediately adjacent mesh points. But, even if $\beta$ is only a more generally located line, then the localized hyperbolas (or lines) which constitute $C_{b}$ or $C_{l}$ can contain points which are of order $h$ away from $\beta$.

In the case of $C_{b}$ this result is the analog of the corresponding case in one space dimension (Section 1) where, if one uses the linear interpolant of the single unit of usable data and an adjacent, unusable unit, then the error in the location of $d$ is not $o(h)$ except in special situations. For, contemplating the possible meshpoint values of $\bar{c}$ associated with a linear border $\beta$, one sees that there exists no mesh square with all four of its corners usable. It follows, as in one dimension, that $C_{b}$ 
cannot be $o(h)$ accurate if any corner is usable (unless two of the $\bar{c}_{i}$ are within $o\left(h^{2}\right)$ of $1 / 2$, or unless two are within $o(h)$ of 1 and the other two are $\left.o(h)\right)$. There can exist three adjacent, usable, and noncolinear $X_{i}$. The analysis of $l$ in this case is more involved (see Counterexample 3.1); the result is that $C_{l}$, too, is not an $o(h)$ accurate approximation to linear borders $\beta$ except in quite special circumstances.

3. Towards Second-Order Approximation. A working principle often yielding second-order accurate approximation is that the algorithm should reproduce linear functions. This is interpreted for the reconstruction problem as the following principle: The algorithm should exactly reconstruct, from associated usable data, all boundaries $\beta$ that are straight lines $L=L(\nu, \Delta)$ with arbitrary unit normal vector $\nu$ (chosen pointing into $\Omega_{1}$ ) and at an arbitrary (signed) distance $\Delta$ measured from some known point along $\nu^{* *}$ The three algorithms to be presented (Sections 7, 11 , and 12) produce only lines $L$, and from local, usable data $\left(X_{i}, \bar{c}\left(X_{i}\right)\right)$; the production of these lines possesses an appropriate invariance under rigid motions and changes in the scale $h$; and two of the three obey our principle. We shall show when these lines provide, in the locale of the data, a second-order accurate approximation to curved borders $\beta$ (see Sections 8, 9, 11, and 12).

But, to continue pursuit of the relative inaccuracy of local linear interpolants: observe that the level curves of the average color function $\bar{c}_{L}$, associated with the $h \times h$ square $S(x, h)$ and the two regions separated by a line $L$, are lines parallel to $L$. Moreover, $\bar{c}_{L}(x)$ is only a function $f_{\nu}(\sigma)$ of the (signed) scaled distance $\sigma:=s / h$ from $x$ to $L$, with $s$, say, as measured along $L$ 's normal, $\nu$. Ambiguity is removed by choosing $s>0$ in $\Omega_{1}$. With this, and no matter how $L$ is oriented, (1) $f_{\nu}$ is strictly monotone increasing where it is neither 0 nor $1 ;(2)$ the symmetry of $S$ means that $f_{\nu}=1 / 2$ exactly when $\sigma=0$, i.e.,

$$
L=\bar{c}_{L}^{-1}(1 / 2) \quad \text { independent of } L \text { and } h
$$

and (3) the graph of $f_{\nu}$ is symmetric under reflection through the point $(0,1 / 2)$. $f_{\nu}$ is called the cumulative distribution function for $\nu$-normal lines (with respect to the centered unit square). It behaves as follows.

If $\nu$ is $( \pm 1,0)$ or $(0, \pm 1)$, then (because a side of $S$ passes over $L_{\nu}$ all at once as $S$ moves along the normal) $f_{\nu}$ is piecewise linear with its nontrivial portion given by $\sigma+1 / 2$ for $|\sigma| \leq 1 / 2$. If $\nu=( \pm \sqrt{2} / 2, \pm \sqrt{2} / 2)$, then (because only a corner of $S$ makes initial contact with $L_{\nu}$ ) $f_{\nu}$ is piecewise quadratic -in fact it is the $C^{1}$ quadratic spline which is identically zero (or one, appropriately) for $|\sigma|>\sqrt{2} / 2$, has knots exactly at $\sigma=0$ and $\pm \sqrt{2} / 2$, and is $1 / 2$ at $\sigma=0$. In the general case, $f_{\nu}$ is also a $C^{1}$ quadratic spline, but with three nonconstant pieces instead of two the middle section is linear with midpoint $(0,1 / 2)$ and represents $\bar{c}_{L}$ when exactly two of the square's corners are on each side of $L$. Summarizing, and more precisely, the cumulative distribution function for $\nu$-normal lines is given by the following: With $\theta(\nu)$ the magnitude of the angle that the line's normal $\nu$ makes with the nearest

${ }^{* *}$ I now know that this requirement was independently expressed in the sixties by DeBar [11] and the seventies by Youngs [28], [29]. Cf. the end of this section. 
coordinate axis (so that $0 \leq \theta(\nu) \leq \pi / 4$ ), and with $\xi(\sigma, \theta):=(\cos \theta+\sin \theta) / 2-|\sigma|$,

$$
f_{\nu}(\sigma)=1 / 2+ \begin{cases}\sigma / \cos \theta, & 0 \leq|2 \sigma| \leq \cos \theta-\sin \theta \\ \left(1 / 2-\xi^{2} / \sin 2 \theta\right) \operatorname{sgn} \sigma, & \cos \theta-\sin \theta \leq|2 \sigma| \\ & \leq \cos \theta+\sin \theta \\ (1 / 2) \operatorname{sgn} \sigma, & |2 \sigma| \geq \cos \theta+\sin \theta .\end{cases}
$$

To verify, as promised in Section 2, that the linear interpolant $l$ of usable adjacent noncolinear meshpoint values of $\bar{c}_{L}$ need yield only a first-order approximation $l^{-1}(1 / 2)=: C_{l}$ to $L$, consider the case that, as $h \rightarrow 0$, the line segment $\overline{X_{i} X_{j}}$ from a usable $X_{i}$ to its usable neighbor $X_{j}$ makes an angle $\theta \neq 0$ with the nearest coordinate axis. With $\bar{c}_{i}:=\bar{c}_{L}\left(X_{i}\right)$, suppose without loss that $\bar{c}_{i}<\bar{c}_{j}$ and (for the moment) that $\bar{c}_{i}<1 / 2<\bar{c}_{j}$. Define $\sigma_{i}$ and $\sigma_{j}$ by

$$
f_{\nu}\left(-\sigma_{i}\right):=\bar{c}_{i}, \quad f_{\nu}\left(\sigma_{j}\right):=\bar{c}_{j} .
$$

Now, along $\overline{X_{i} X_{j}}, l$ varies linearly from $\bar{c}_{i}$ to $\bar{c}_{j}$; so (on $\overline{X_{i} X_{j}}$ )

$$
l^{-1}(1 / 2)=X_{i}+\left(X_{j}-X_{i}\right)\left(1 / 2-\bar{c}_{i}\right) /\left(\bar{c}_{j}-\bar{c}_{i}\right) .
$$

However, as the level lines of $\bar{c}$ are parallel to $L, \bar{c}$ itself varies in concert with $f_{\nu}(3.2)$ : for $x$ in $\overline{X_{i} X_{j}}$,

$$
\bar{c}(x)=f_{\nu}\left(-\sigma_{i}+\left(\sigma_{j}+\sigma_{i}\right)\left\|x-X_{i}\right\| /\left\|X_{j}-X_{i}\right\|\right) .
$$

Now, the graph of $f_{\nu}$ is symmetric under reflection through $(0,1 / 2)$ : for $\sigma \geq 0$,

$$
f_{\nu}(\sigma)-1 / 2=: g(\sigma)=1 / 2-f_{\nu}(-\sigma)
$$

(note $g(0)=0, g$ is differentiable with $D g$ nonincreasing, and $D^{2} g$ is piecewise constant). Using the graph of $f_{\nu}$, one finds that on $\overline{X_{i} X_{j}}$, and with $g_{i}:=g\left(\sigma_{i}\right)$ and $g_{j}:=g\left(\sigma_{j}\right)$,

$$
\left|\bar{c}^{-1}(1 / 2)-l^{-1}(1 / 2)\right|=h \sigma_{i} \sigma_{j}\left|g_{i} / \sigma_{i}-g_{j} / \sigma_{j}\right| /\left(g_{i}+g_{j}\right) .
$$

Consequently, if either $\bar{c}_{i}$ or $\bar{c}_{j}$ lies strictly away from the linear portion of $f_{\nu}$, i.e., if

$$
\min \left(\bar{c}_{i}, \bar{c}_{j}\right)<(\tan \theta) / 2+o(h), \text { or } \max \left(\bar{c}_{i}, \bar{c}_{j}\right)>1-(\tan \theta) / 2+o(h),
$$

and if

$$
\bar{c}_{j}+\bar{c}_{i}-1\left(=g_{j}-g_{i}\right) \neq o(h),
$$

then the error in locating the intersection of $L$ with $\overline{X_{i} X_{j}}$, using $l^{-1}(1 / 2)$, is not $o(h)$.

This result is unaltered if $\bar{c}_{i}$ and $\bar{c}_{j}$ lie on the same side of $1 / 2$.

Consider, now, the case when three vertices of a mesh square $X_{1} X_{2} X_{3} X_{4}$ are usable-without loss, assume $\left(X_{4}, \bar{c}_{4}\right)$ is unusable and $\bar{c}_{4}=0$-and let $S_{i}$ be the $h \times h$ square centered on $X_{i}$ with $\bar{X}$ the point common to all four $S_{i}$. Since $L$ intersects the interior of all squares but $S_{4},\left(\sigma_{2}, \bar{c}_{2}\right)$ lies in the nonlinear portion of $f_{\nu}$, and $\bar{c}_{2}>1 / 2$. There are a number of cases to consider. Suppose, (1) that $\bar{c}_{2}=1-o(h)$, i.e., $L$ is within $o(h)$ of $\bar{X}$. The only way for $(3.3 \mathrm{~b})$ to fail is that 
$\theta=o(h)$; i.e., $L$ becomes a parallel to a coordinate axis lying halfway between meshpoints; this is one of the exceptional cases when $l^{-1}(1 / 2)$ can approach $L$ within $o(h)$. Now, suppose $\bar{c}_{2} \neq 1-o(h)$. For (3.3b) to fail for $j=2$ and $i=1$ or 3 , $L$ must cut the rectangle $S_{i} \cup S_{2}$ into two trapezoids of nearly equal area. For this to be so for both $i=1$ and 3 , (2) $\theta$ must approach $\pi / 4$ while $L$ again comes close to lying halfway between meshpoints. This is a second exception. So, now suppose (3.3b) is satisfied for $j=2$ and some $i$. For $\bar{c}_{2}$ to approach the linear portion of $f_{\nu}$, some point of $L$ must approach the triangular half of $S_{2}$ opposite $\bar{X}$. If more than one point does, we have case (2) again. If only one point does-say, between $S_{2}$ and $S_{1}$-then $\bar{c}_{1}$ approaches zero. But, $L$ cannot approach $\bar{X}$ - that would be exception (1), so $\bar{c}_{1}$ is in the other nonlinear portion of $f_{\nu}$ and is not within $o(h)$ of its linear portion. This concludes the proof of

COUNTEREXAMPLE 3.1. Unless a linear border $\beta=L$ comes close to lying halfway between meshpoints parallel to a side of a mesh square or to its diagonal, the linear interpolant $l$ of three usable values of $\bar{c}$ on a mesh square (the fourth being necessarily unusable) yields a line $C_{l}:=l^{-1}(1 / 2)$ whose points in the mesh square are not all within $o(h)$ of $L$.

But, in the remaining case of usable data $\left(X_{i}, \bar{c}_{i}\right)$ associated with a linear border $\beta=L$, local linear interpolation assists in exactly reproducing $L$-indeed, this case corresponds to the use of the single unit of usable data in one dimension. The data for this case satisfy: on two adjacent mesh squares, the two common meshpoints $X_{1}$ and $X_{2}$ are usable while the remaining four are not. So, since $L$ passes out of the rectangle $S_{1} \cup S_{2}$ through its short sides, both $S_{1}$ and $S_{2}$ have a pair of corners on either side of $L$. Thus, $\bar{c}$ itself is linear on the line segment $\overline{X_{1} X_{2}}$-and, so, between $\bar{c}_{i}$ and $1 / 2$ on any line through $X_{i}, i=1$ (or $i=2$ ); in particular, on the line normal to $L$. Consequently, $L$ can be pinned down almost uniquely as follows.

For now, according to the top line of (3.2) and for $i=1$ or 2 , the distance of $L=L_{\nu}$ from $X_{i}$ is $h\left|\bar{c}_{i}-1 / 2\right| \cos \theta(\nu)=: r_{i}(\nu)$. This means that, for each (now unknown) $\nu$, the only possible candidates are one of the up to four lines-each with its two normals---tangent to these two circles; and the signs of the $\bar{c}_{i}-1 / 2$ reduce these line/normal candidates to two (see Section 7 and Appendix 2). As a border, one of these lines will yield the given $\bar{c}_{i}$ as its average color at the two $X_{i}$ if and only if its normal makes the angle $\theta$ with a coordinate axis. So: one guesses a normal, determines the two radii, selects the more compatible mutually tangent line with its normal, and iterates appropriately.

This exemplifies the fact that one may, in complete analogy with the onedimensional case, (almost) uniquely locate a linear border relative to any "usable" line segment $\overline{X_{1} X_{2}}$, i.e., any segment such that the data $\left(X_{1}, \bar{c}_{1}\right)$ and $\left(X_{2}, \bar{c}_{2}\right)$ are both usable. One does not approximate $\bar{c}$ on $\overline{X_{1} X_{2}}$ to do this; one assumes (3.2) holds exactly (suitably stretched) on the segment for some $\nu$, and one both determines $L$ 's distance from the $X_{i}$ and orients $L$ with respect to the line segment directly from the data. There will generally be two lines compatible with two usable data--indeed, the symmetries involved here imply reflection of a solution (with its normal) in the line containing $\overline{X_{1} X_{2}}$ will be the other solution but a third datum allows the appropriate choice. Again, see Section 7 and Appendix 2. 
So, the apt notion seems (and will prove) to be not that three points determine a plane - and so a surface approximating the average color function near the border, from which the border itself may be estimated-but that two points, albeit blurred to circles, directly determine a line locally approximating the border.

I have subsequently become aware that DeBar [11] notes and uses the fact that the areas a line cuts from two adjacent mesh squares (almost) determine that lineand that this also comprises the kernel of Youngs' [28], [29] algorithm. Given the average color of each cell in a $3 \times 3$ pile of congruent rectangular cells, Youngs associates a line with the central cell (presumed usable) as follows. For each of its eastern, western, northern and southern neighbors in turn (and if usable), he constructs the intersection of the line yielding the two average colors - choosing between the two possible solutions using other average colors - with the cells' common edge (the exceptional case of intersection near a corner is treated with sufficient accuracy). It is apparent from Youngs [27] that he could have constructed the line itself. It is particularly striking that this kernel of Youngs' procedure [27] does not invoke lines mutually tangent to circles or the iterations we propose for this case-it is direct and explicit. Youngs goes on to mold all this information into an algorithm by determining, from these intersections, the normal $\nu$ to the line associated with the central cell; and then, from $\nu$ and this cell's average color, the line itself.

4. Other Scaled Averages. The use of a grid-size square in defining the "average color" function $\bar{c}(1.2)$ (of a color function $c$ ) led to various difficulties in reproducing linear borders from its meshpoint values. We now consider more general averages, some of which will ease these difficulties.

Assume one is given a probability density $\rho$ defined on the plane. Redefine the "average color" of a color function $c$ to be the scaled convolution

$$
\bar{c}(x):=\iint_{\text {plane }} \rho((\xi-x) / h) c(\xi) d A(\xi) / h^{2} ;
$$

it bears the scale $h$ as a parameter. Associated with $\rho$ is its cumulative distribution function for $\nu$-normal lines,

$$
f_{\nu}(\sigma):=\int_{-\sigma}^{\infty} \int_{-\infty}^{\infty} \rho(s \nu+t \omega) d t d s, \quad \omega \cdot \nu=0,\|\omega\|=\|\nu\|=1 .
$$

Recall that when the border is a linear border, $L=L_{\nu}, \nu$ points into $\Omega_{1}$-i.e., towards that side of $L$ where $c=1$ - so that still, as above $(3.1), \bar{c}_{L}(x)=f_{\nu}(\sigma)$, with $h \sigma$, the signed distance from $L$ to $x$ as measured along $\nu$, being negative for $x$ in $\Omega_{0}$ and nonnegative, otherwise. Concerning the present analog of (3.1) itself: for $L=L_{\nu}$ to coincide with $\bar{c}_{L}^{-1}\left(f_{\nu}(0)\right)$ (and not be just a proper subset), one must assume that $f_{\nu}$ is strictly monotone at $\sigma=0$. We shall, in fact, impose a stronger extension of this: let $M_{0}(\sigma, \nu):=\left(\partial f_{\nu} / \partial \sigma\right)(\sigma, \nu)$ be the mass of the line (induced by the restriction to it of $\rho$ ) which is normal to $\nu$ and passes through the point $\left(-\sigma \nu_{x},-\sigma \nu_{y}\right)$. Then we assume, for each $\nu$, that the interior of the support of $M_{0}(\cdot, \nu)$ is a nontrivial interval which contains 0 and on which $M_{0}(\cdot, \nu)$ is positive. In particular, then, $f_{\nu}(0)>0$; and $f_{\nu}(0)=1 / 2$ if $\rho$ is symmetric under reflection through the origin. 
The use of general densities can lead to the same type of orientation problems that the square led to. What is the simplest which has no orientation problems? It is the characteristic function of a circular disc.

5. Using the Average Color of a Disc. With $\rho$ given by $4 / \pi$ times the characteristic function of a circular disc centered on the origin and of diameter one, we use (4.1) to define the "disc-average" color function $\bar{c}$ of a color function $c$. This average color also has the properties noted in the paragraph containing (3.1). But, now, the associated "cumulative distribution function for $\nu$-normal lines $L$ " is independent of $L$ 's orientation, i.e., of $\nu$, and is given by

$$
f(\sigma)=1 / 2+ \begin{cases}\left(\sin ^{-1}(2 \sigma)+4 \sigma \sqrt{1-4 \sigma^{2}}\right) / \pi, & |\sigma| \leq 1 / 2 \\ \operatorname{sgn} \sigma / 2, & |\sigma| \geq 1 / 2\end{cases}
$$

At each joint $\sigma_{0}$ with its constant pieces it deviates by $(4 / \pi)\left|\sigma-\sigma_{0}\right|^{3 / 2}(1+o(1))$ from being constant-this represents a compromise between the two possible behaviors of (3.2) at those joints.

$f(5.1)$ is incorporated into an algorithm to reproduce lines $L$ from nontrivial meshpoint values of $\bar{c}$ as follows. From the two usable values, $\bar{c}_{1}=\bar{c}_{L}\left(X_{1}\right)$ and $\bar{c}_{2}=\bar{c}_{L}\left(X_{2}\right)$, find $\sigma_{1}:=f^{-1}\left(\bar{c}_{1}\right)$ and $\sigma_{2}:=f^{-1}\left(\bar{c}_{2}\right)$. Then (see Section 7 for details), $L$ is one of the (up to four) lines simultaneously tangent to the two circles $C_{i}(i=1$ and 2$)$ centered on $X_{i}$ and of radius $r_{i}:=h\left|\sigma_{i}\right|$. (Here, $h$ is the scale parameter utilized in (4.1) - in this case the diameter of the circle one chooses to average over.) The reflection of such an $L$ (with its normal) in the line containing the segment $\overline{X_{1} X_{2}}$ is a second solution (if distinguishable from $L$ ). Of the up to eight line/normal possibilities, at most two are solutions; and the choices are easily made using $\bar{c}_{1}$ and $\bar{c}_{2}$. The value of $\bar{c}_{L}\left(X_{3}\right)$ at almost any third point $X_{3}$ discriminates between these two solutions.

However, if the usable values $\bar{c}_{i}$ are not known in advance to be $\bar{c}_{L}\left(X_{i}\right)$ for some line $L=L_{\nu}$, then there may exist no $L_{\nu}$ such that $\bar{c}_{L}\left(X_{i}\right)=\bar{c}_{i}$ for $i=1$ and 2. For, the required change $\left|\bar{c}_{2}-\bar{c}_{1}\right|$ may be too large for the average coloring $\bar{c}$ to accomplish it in the given distance $\left\|X_{2}-X_{1}\right\|$-even if $L$ were perpendicular to $\overline{X_{1} X_{2}}$. This is also easy to decide; see Section 7 , especially Summary 7.1. In practice, one would use two usable values of $\bar{c}$ associated with a smooth border $\beta$-values bounded away from both zero and one and at two points of order $h$ apart - to determine a pair of lines. The appropriate one of the pair can be and is selected on the basis of a third value of $\bar{c}$ nearby. Then, if $\overline{X_{1} X_{2}}$ is not nearly normal to the border $\beta$, any portion of this line in any $O(h)$ neighborhood of the two points will be within $O\left(h^{2}\right)$ of $\beta$ (Propositions 8.2 and 9.1).

The same sort of algorithm works for any probability density $\rho$ (Section 4 ) which is azimuthally symmetric, i.e., is a function only of $r$ in polar coordinates centered on the origin. The new associated cumulative distribution function for lines $f$ (4.2) replaces (5.1) in the algorithm just described (for more detail, see Algorithm 7.1 below). Since the derivative of $f$ is bounded away from zero except at the extremities of $f$ 's usable interval (see the end of Section 4), this algorithm will also yield locally second-order accurate approximation to curved borders in the circumstances just described. 
It can be computationally costly to repeatedly invert even a strictly monotone function $f$, either analytically or numerically, unless $f$ is linear. How could this last be arranged?

6. Discs Suitably Sensitive to Lines. Let us see if we can make the disc sensitive, in some fashion, so that the associated cumulative distribution function for lines $f$ is linear where it is neither zero nor one:

$$
f(\sigma)=1 / 2+ \begin{cases}\sigma, & |\sigma| \leq 1 / 2 \\ \operatorname{sgn} \sigma / 2, & |\sigma| \geq 1 / 2\end{cases}
$$

Then, $f$ would coincide with the original $f_{\nu}$ of (3.2) when $L$ was parallel to a side of the square $S$ we then averaged over (see (3.2) with $\theta=0$ ). The use of such an $f$ in the algorithm of Section 5 would be particularly easy, since (now) $\sigma_{i}=\bar{c}_{i}-1 / 2$. Moreover, there would exist a line $L$ with $\bar{c}_{L}\left(X_{i}\right)=\bar{c}_{i}(i=1$ and 2 both usable) if and only if $\left|\bar{c}_{2}-\bar{c}_{1}\right| \leq\left\|X_{2}-X_{1}\right\| / h$ (see Summary 7.1). And, there would exist exactly two such lines (each the reflection-with its normal-of the other in the line containing $\overline{X_{1} X_{2}}$ ) if and only if the inequality is strict (again, see Section 7 ). The local approximation properties of lines locally determined from local usable data associated with this special disc-averaging, data further restricted as in Section 5, would be as there described.

This special density, $\rho$, will be azimuthally symmetric; and, nonzero only on the disc of diameter one which is centered at the origin. Now, unlike the square just encountering a line $L$ parallel to its side, the disc bends away from $L$. Hence, $\rho(r)$, $r=\sqrt{x^{2}+y^{2}}$, will have to be quite large at the circumference $r=1 / 2$ of the disc; in fact, it becomes infinite there like $1 / \sqrt{1 / 2-r}$. To see this, note that the rate of change of $f(6.1)$ is the amount of $\rho$ on a line perpendicular to a radius and a distance $\sigma$ from the disc's center: for $0 \leq \sigma<1 / 2$,

$$
(d f / d \sigma)(\sigma)=1=2 \int_{0}^{\sqrt{1 / 4-\sigma^{2}}} \rho\left(\sqrt{\sigma^{2}+z^{2}}\right) d z .
$$

One easily verifies that the solution of $(6.2)$ is

$$
\rho(r):=1 /\left(\pi \sqrt{1 / 4-r^{2}}\right), \quad 0 \leq r<1 / 2 .
$$

Since $\rho(r) \geq 0$ and the amount of $\rho$ on the half-disc is $\int_{0}^{1 / 2} 1 d \sigma, \rho$ is indeed a probability distribution on the disc.

The integral equation (6.2) was not solved by inspection. It happens that (6.2) also occurs in the determination, from X-ray observations experimentally specifying a (more general) function $d f / d \sigma$ above, of an azimuthally symmetric but otherwise unknown density distribution $\rho$ in a solid ball (e.g., Carter, Pimbley, and Wing [7], Wing [26]). In this connection, Carter, Pimbley, and Wing [7] and, especially, Faber and Wing [13] record the judicious set of variable changes transforming (6.2) into Abel's integral equation.

More generally: if it is determined-say, experimentally - that a visual system is able to most accurately associate a line with a prescribed blurred version $f$ different from (6.1) (but still independent of orientation), then one can solve the correspondent (6.2) for the associated azimuthally symmetric sensitivity $\rho_{f}$. 
7. Locating Linear Borders Relative to a Usable Line Segment. The usable data $\left(X_{1}, \bar{c}_{1}\right),\left(X_{2}, \bar{c}_{2}\right), X_{1} \neq X_{2}$, is given. Let $\tau$ be the unit tangent vector for the line segment $\overline{X_{1} X_{2}}$, directed from $X_{1}$ to $X_{2}$; and let $\delta:=\left\|X_{2}-X_{1}\right\|$. Let $L=L_{\nu}$ be the unknown linear border with its normal $\nu$ pointing into $\Omega_{1}$, and let $h$ be the scale for the color blurring (1.2) or (4.1). Let $f_{\nu}$ be the associated cumulative distribution function for $\nu$-normal lines (see (3.2), (4.2), (5.1), or (6.1)). Then, since the level lines for $\bar{c}_{L}$ are parallel to $L, \bar{c}_{L}$ varies along $\overline{X_{1} X_{2}}$ in concert with $f_{\nu}$ :

$$
\bar{c}\left(X_{1}+s \tau\right)=f_{\nu}\left(f_{\nu}^{-1}\left(\bar{c}_{1}\right)+(s / h) \cos L_{\tau, \nu}\right) ;
$$

here $L_{\tau, \nu}$, the angle from $\tau$ to $\nu$, lies in $[0,2 \pi)$. Taking $s=\delta$, we obtain

$$
\cos L_{\tau, \nu}=h\left(f_{\nu}^{-1}\left(\bar{c}_{2}\right)-f_{\nu}^{-1}\left(\bar{c}_{1}\right)\right) / \delta .
$$

This is to be solved for its single unknown, $\nu$; and the associated line $L_{\nu}$ is to be then located.

The case that $f_{\nu}=f$ independent of $\nu$ - for example, that $\rho$ (4.1) is azimuthally symmetric -is instructive. Then, (7.2) has a solution if and only if

$$
h\left|f^{-1}\left(\bar{c}_{2}\right)-f^{-1}\left(\bar{c}_{1}\right)\right| / \delta \leq 1
$$

and, if it has one solution, then it has precisely two (having the same values of $\left.\cos L_{\tau, \nu}\right)$ unless $L_{\tau, \nu}$ is 0 or $\pi$.

More detail is seen from the geometry of solutions, which is as follows. For $i=1$ and 2 , set

$$
r_{i}(\nu):=h\left|f_{\nu}^{-1}\left(\bar{c}_{i}\right)\right|
$$

and let

$$
C_{i}(\nu) \text { be the circle of radius } r_{i}(\nu) \text { centered on } X_{i} \text {. }
$$

Then $L_{\nu}$ solves (7.2) only if it is one of the lines (if any) which are simultaneously tangent to both $C_{i}(\nu)$. From this, if $f_{\nu}=f$ is independent of $\nu$, the reflection of a solution (including its normal $\nu$ ) in the line containing $\overline{X_{1} X_{2}}$ is also a solution. Moreover, then, if $X_{i}, i=1,2,3$, are usable and if the associated $\bar{c}_{i}$ are, in fact, $\bar{c}\left(X_{i}\right)$ for some line, then the pair of lines solving (7.2) can be distinguished using the (signed) distance $h f_{\nu}^{-1}\left(\bar{c}_{3}\right)$ that the true solution is from $X_{3}$ if and only if the three $X_{i}$ are not colinear.

If the two $\bar{c}_{i}$ used are not known to be $\bar{c}_{L}\left(X_{i}\right)$ for the same line $L$, then existence of solutions to (7.2) becomes a problem. Let us first suppose that $f_{\nu}=f$ is independent of $\nu$. If $\bar{c}_{1}=1 / 2(=f(0))=\bar{c}_{2}$, then the only two solutions are the line through $\overline{X_{1} X_{2}}$, using either normal. Otherwise, the only possibilities for solutions are the lines simultaneously tangent to the two circles $C_{i}$ of radius $r_{i}$ (now independent of $\nu$ ). The necessary orientation of a solution's normal then becomes determinative as follows. (1) If $\delta<\left|r_{2}-r_{1}\right|$, then there are no possibilities or solutions, since the smaller disc lies inside the larger. (2) If $\delta=\left|r_{2}-r_{1}\right|$, then the smaller is internally tangent to the larger, and there is one line constituting two possibilities (for the two possible normals). Now use the fact that $\nu$ (if located on a potential solution) points away from $X_{i}$ if $\bar{c}_{i}<1 / 2$, towards $X_{i}$ if $\bar{c}_{i}>1 / 2$, and 
either way if $\bar{c}_{i}=1 / 2$. This yields: (2a) If the $\bar{c}_{i}$ do not lie on different sides of $1 / 2$, there is exactly one solution; and $(2 \mathrm{~b})$ if the $\bar{c}_{i}$ lie on different sides of $1 / 2$, there are no solutions. (3) If $\left|r_{2}-r_{1}\right|<\delta<r_{2}+r_{1}$, then the discs' interiors overlap, and there are two lines representing four possibilities. Among them, (3a) If the $\bar{c}_{i}$ do not lie on different sides of $1 / 2$, there are exactly two solutions; and (3b) if the $\bar{c}_{i}$ lie on different sides of $1 / 2$, there are no solutions. If (4) $\delta=r_{1}+r_{2}$, the two circles are (externally) tangent: we have (4a) two solutions as before, but in case (4b) there is exactly one solution. If (4) $\delta>r_{1}+r_{2}$, we have exactly two solutions.

SUMMARY 7.1. For $f_{\nu}=f$ independent of $\nu:$ If the $\bar{c}_{i}$ lie on the same side of $1 / 2$, then there exists an oriented line $L_{\nu}$ mutually tangent to the two circles (7.3) and solving (7.2) if and only if $\delta \geq\left|r_{2}-r_{1}\right|$. If the $\bar{c}_{i}$ lie on different sides of $1 / 2$, then there is a solution if and only if $\delta \geq r_{2}+r_{1}$. The mirror image of a solution (with its normal) in the line containing $\overline{X_{1} X_{2}}$ is also a solution.

Proposition 8.1 and Appendix 2 consider the existence question for $\nu$-dependent $f_{\nu}$.

The following algorithm includes a crude but effective means of distinguishing between solutions when $f_{\nu}$ is independent of $\nu$.

AlgorithM 7.1 Given the usable data $\left(X_{1}, \bar{c}_{1}\right),\left(X_{2}, \bar{c}_{2}\right)$, with $0 \neq\left\|X_{1}-X_{2}\right\|=$ $O(h)$; take the origin to be a vertex of the square opposite its diagonal $\overline{X_{1} X_{2}}$. Suppose first that $f_{\nu}$ is independent of $\nu$. Then

(1) For $i=1,2$, compute $\sigma_{i}:=f_{\nu}^{-1}\left(\bar{c}_{i}\right)$ and $a_{i}:=h \sigma_{i}$.

(2) Use Algorithm A1.2 in Appendix 1 with $a:=\left(a_{1}, a_{2}\right)^{T}$ to determine the up to two lines $L\left(\nu_{j}, \Delta_{j}\right)$, if any, mutually tangent to the circles $(7.3 \mathrm{~b})$ and solving (7.2).

(3) If there are two solutions, choose, as the line $L_{A}$ produced by this algorithm, the one compatible with an unusable data pair $\left(X_{3}, \bar{c}\left(X_{3}\right)\right)$, with $X_{3}$ not within $O(h)$ of the interior of the vertical angle containing $\overline{X_{1} X_{2}}$.

We offer no specific algorithm when $f_{\nu}$ depends on $\nu$, although a one-variable Newton's method could be tried on (7.2), based on (8.6)-(8.8). We simply suppose that one can find a line $L_{A}$, with normal $\nu$, satisfying (7.2)-(7.3), i.e., (1)-(3) above.

The associated map $\mathscr{P}:\{$ borders $\beta\} \rightarrow$ linear borders $\}$ (given by evaluating the average color at $X_{1}$ and $X_{2}$, applying the algorithm, and defining the new border using $L_{A}$ and its normal) is a projection (since $\mathscr{P}(\mathscr{P})=\mathscr{P}$ ) onto the linear borders sufficiently near $X_{1}$ and $X_{2}$ for both to be usable. The linear border it produces is the interpolant, should any exist among such linear borders, of the pair of functionals consisting of evaluation of the average color at the two usable points $X_{1}$ and $X_{2}$. As seen in Summary 7.1, such an interpolant need not exist for an arbitrary nonlinear border which is usable at $X_{1}$ and $X_{2}$. (The projector associated with the algorithm of Section $12 \cdots$ and the operator, of Section 11 need not yield interpolants of those algorithms' triples of functionals.)

8. Stability; and Convergence to the Border. The border $\beta$ between $\Omega_{0}$ and $\Omega_{1}$ in (1.1) has bounded curvature and does not cross itself. Let $\bar{c}$ be the average color function with scale $h((1.2)$ or $(4.1))$ of the color function $c$ (1.1) 
associated with $\Omega_{0}$ and $\Omega_{1}$. We assume $h \rightarrow 0$. Let $X_{\beta}$ be a fixed point on $\beta$. Let $X_{1} \neq X_{2}$ be two usable points converging to $X_{\beta}$ at least as fast as $h$ goes to 0 ; i.e., suppose $\left\|X_{i}-X_{\beta}\right\|=O(h)$ with $\bar{c}_{i}:=\bar{c}\left(X_{i}\right)$ strictly between zero and one, $i=1,2$ (so, in particular, $\left.\delta:=\left\|X_{2}-X_{1}\right\|=O(h)\right)$. Let $L_{\beta}=L\left(\nu_{\beta}, \Delta_{\beta}\right.$ ) be the line tangent to $\beta$ at $X_{\beta}$ with normal $\nu_{\beta}$ pointing into $\Omega_{1}$ and at the (signed) distance $\Delta_{\beta}$ from $X_{1}$ as measured along $\nu_{\beta}$. Algorithm 7.1 associates lines $L_{A}=L(\nu, \Delta)$ with data sets $\left\{\left(X_{i}, \bar{c}_{i}\right), i=1,2\right\}$. The goal for this section is to discover when $\nu \rightarrow \nu_{\beta}$ and $\Delta \rightarrow \Delta_{\beta}$ at rates $O\left(h^{2} / \delta\right)$ and $O\left(h^{3} / \delta\right)$, respectively; and when $L_{A}$ is within $O\left(h^{3} / \delta\right)$ of $\beta$ throughout any neighborhood of $X_{\beta}$ of diameter $O(h)$. Propositions 8.1 and 8.2 will impose further restrictions-on the directions $\tau$ of the line segments $\overline{X_{1} X_{2}}$, on the $\bar{c}_{i}$-which allow this conclusion.

Since the average color near $X_{\beta}$ differs in a small, estimable way from the average color associated with two regions separated there by $L_{\beta}$ instead (Remark 8.1 below), and since Algorithm 7.1 reproduces lines and so would reproduce $L_{\beta}$ from data taken from the latter average coloring, we begin with a stability result, for linear borders, against perturbations in the algorithm's data. For this we shall impose a modified definition of "usable" data:

Definition 8.1. The usable numbers $\bar{c}(X)$ are "uniformly usable" means that the slope of $f_{\nu}^{-1}$ is bounded uniformly over the smallest interval containing them and for all $\nu$.

For example: "usable" implies "uniformly usable" for (6.1), but not for (5.1) or (3.2), when "uniformly usable" would refer instead to numbers between usable limits fixed a priori - say, 0.02 and 0.98. When $f_{\nu}=f$ is independent of $\nu$ (Section 7 ), our algorithm is clearly less stable if the two circles, determining $L_{A}$ by its mutual tangency to them, are close to mutual tangency themselves. When $f_{\nu}$ really depends on $\nu$, there is a different, but still "rarely" satisfied condition, determinable a posteriori, on data pairs which can render stability near them completely opaque. This is part of the content of

PROPOSITION 8.1. (Concerning the stability of Algorithm 7.1.) Let the border $\beta$ be a line (so that $L_{A}=L_{\beta}=\beta$ ). Suppose, for $i=1,2$, that $(a) \bar{c}_{i}=\bar{c}_{L_{\beta}}\left(X_{i}\right)$ and its perturbation $\tilde{c}_{i}$ are uniformly usable, and $(b)\left|\tilde{c}_{i}-\bar{c}_{i}\right|=O(\varepsilon), \varepsilon=o(1)$. Set $\alpha:=L_{\tau, \nu}$. Should one exist, let $\tilde{L}=L(\tilde{\nu}, \tilde{\Delta})$ be a line yielded by Algorithm 7.1 for the data $\left(X_{i}, \tilde{c}_{i}\right), i=1,2$.

Suppose first that (1) $f_{\nu}$ is independent of $\nu$. Then $\tilde{L}$ is unique. If $\sin \alpha \neq o(1)$, then $\tilde{L}$ exists for $h \varepsilon / \delta$ small enough, and

$$
\left\|\tilde{\nu}-\nu_{\beta}\right\|=O(h \varepsilon / \delta) .
$$

Otherwise, should $\tilde{L}$ exist, then

$$
\left\|\tilde{\nu}-\nu_{\beta}\right\|=O(\sqrt{h \varepsilon / \delta})
$$

In either case,

$$
\left|\tilde{\Delta}-\Delta_{\beta}\right|=O(h \varepsilon) .
$$

However, if (2) $f_{\nu}$ depends on $\nu$, then a sufficient condition for local existence and stability of $\tilde{\alpha}$, i.e., $\tilde{\nu}$, is the more general condition

$$
\cos \alpha\left(\mu_{2}^{(1)}-\mu_{1}^{(1)}\right) /\left(\sigma_{2}-\sigma_{1}\right)-\sin \alpha \neq o(1),
$$


where $\mu_{i}^{(1)}:=\mu^{(1)}\left(\sigma_{i}, \nu\right), \sigma_{i}:=f_{\nu}^{-1}\left(\bar{c}_{i}\right)$, and $\mu^{(1)}(\sigma, \nu)$ is $\rho$ 's "specific first moment for $\nu$-normal lines," described at (8.7)-(8.8) below and presumed to have a firstorder $\sigma$-difference quotient uniformly bounded on relevant triples $\left(\sigma_{1}, \sigma_{2}, \nu\right)$. With (8.4), $\tilde{L}$ exists and is unique when $h \varepsilon / \delta$ is small enough; and convergence is governed by (8.1) and

$$
\left|\tilde{\Delta}-\Delta_{\beta}\right|=O\left(h^{2} \varepsilon / \delta\right) \text {. }
$$

Proof. Suppose (1) $f_{\nu}=f$ is independent of $\nu$. For (8.3): the (signed) distances $\Delta_{A}=\Delta_{\beta}$ of $L_{A}=L_{\beta}$ from $X_{1}$ are both $h f^{-1}\left(\bar{c}_{1}\right)$; while the distance $\tilde{\Delta}$ of $\tilde{L}$ from $X_{1}$ is $h f^{-1}\left(\tilde{c}_{1}\right)$ (7.3a) as a function of $\bar{c}=\tilde{c}_{1}$ on the interval of uniform usability. This function is Lipschitz continuous with constant bounded by $h$ times the slope of $f^{-1}$ on that interval. Similarly for (8.1) and (8.2): the arccosine of the right side of (7.2) is likewise Lipschitz if $\sin \alpha$ is bounded away from zero; otherwise, its modulus of continuity is the square root function.

If (2) $f_{\nu}$ depends on $\nu$, we apply the implicit function theorem to (7.2) in the form

$$
F\left(\alpha, \bar{c}_{1}, \bar{c}_{2}\right):=\cos \alpha-h\left(f_{\nu(\alpha)}^{-1}\left(\bar{c}_{2}\right)-f_{\nu(\alpha)}^{-1}\left(\bar{c}_{1}\right)\right) / \delta=0 \quad\left(\alpha:=L_{\tau, \nu}\right),
$$

computing $\partial F / \partial \alpha$ by invoking $f_{\nu(\alpha)}^{-1}\left(f_{\nu(\alpha)}(\sigma)\right) \equiv \sigma$ to find that

$$
-\partial f_{\nu(\alpha)}^{-1} / \partial \alpha=\left(\partial f_{\nu(\alpha)} / \partial \alpha\right) /\left(\partial f_{\nu(\alpha)} / \partial \sigma\right)=: \mu^{(1)}
$$

With this, and since $F=0$ at the data for $L_{\beta},(8.4)$ includes $\partial F / \partial \alpha \neq 0$ there. We conclude existence and uniqueness of $\tilde{\alpha}=\alpha\left(\tilde{c}_{1}, \tilde{c}_{2}\right)$ for nearby data, for which (8.4) leads to (8.1). Knowing $\mu^{(1)}$, Newton's method (for one variable) could be tried on (8.6). With $f_{\nu}$ given by (4.2), the meaning of the associated quantities in $\mu^{(1)}(\sigma, \nu)$ (8.7) is clear: the denominator is the mass $M_{0}(\sigma, \nu)$ (see the end of Section 4) of the line (with linear density $\rho$ ) normal to $\nu$ through the point

$$
P_{\sigma, \nu}:=\left(-\sigma \nu_{x},-\sigma \nu_{y}\right)
$$

while the numerator is proportional to the first moment of that line about $P_{\sigma, \nu}$ (in which mass points to the left of $\nu$ receive the positive weights). The content of (8.4) is less clear in general ${ }^{* * *}$; except that there is a two-dimensional set in $\left(\alpha, \bar{c}_{1}, \bar{c}_{2}\right)$ space which one must avoid in order to conclude it, and hence (8.1), when $f_{\nu}$ truly depends on $\nu$. The extent to which stability is destroyed near the set where (8.4) fails is unclear - in contrast to the situation leading to (8.2). Inverting $f_{\tilde{\nu}}$ instead of $f_{\nu_{\beta}}$ gives (8.5). $\rho$ 's "specific second moment for $\nu$-normal lines" occurs in more precise error estimates for approximate normals (9.6) and in the determination of approximate curvatures (Sections 9-10).

***(November, 1987) If $\rho$ is constant on its support, then (8.4) holds if and only if $\cos \alpha\left(\Delta l_{2}-\Delta l_{1}\right) /(2 d)-\sin \alpha \neq o(1)$ with $\Delta l_{j}$ the difference between the lengths of the left and right portions of the lines $l_{j}(8.8)$ and $d$ the signed distance along $\nu$ from $l_{2}$ to $l_{1}$. In particular, it follows that the line produced by applying Algorithm 7.1 to the average color $\bar{c}(1.2)$ of two usable, interiorly disjoint $h \times h$ squares having rectangular union $R$ - i.e., the line associated with DeBar's [11] algorithm (and the kernel of Youngs' [28], cf. Section 3) is stable (and so is locally second-order accurate for smooth curved borders when the data is "usefully usable," cf. Proposition 8.2). The trickiest verification is when the linear border crosses both a short and a long side of $R$. 
We now estimate the extent to which the data, i.e., the average color $\bar{c}\left(X_{i}\right)$, $i=1,2$ (4.1) (of the coloring (1.1) associated with $\Omega_{0}, \Omega_{1}$, and $\beta$ ) are perturbations of the values at the $X_{i}$ of the average color $\bar{c}_{L_{\beta}}$ (of the coloring associated with (4.1) and the line $L_{\beta}$ tangent to $\beta$ at $X_{\beta}$, i.e., normal to $\nu_{\beta}$ and tangent to $\tau_{\beta}$ ). We use the linearity (in $c$ ) of the scaled convolution (4.1) and the properties of the scaling to find that

$$
\begin{aligned}
\left|\left(\bar{c}-\bar{c}_{L_{\beta}}\right)\left(X_{i}\right)\right|=\left|\iint \rho\left(\left(\xi-X_{i}\right) / h\right)\left(c-c_{L_{\beta}}\right)(\xi) d A(\xi) / h^{2}\right| \\
=\left|\iint \rho(a+x)\left(c-c_{L_{\beta}}\right)\left(X_{\beta}+h x\right) d A(x)\right|, \quad a:=\left(X_{\beta}-X_{i}\right) / h \\
\quad \leq \iint \rho(a+x)\left|c-c_{L_{\beta}}\right|\left(X_{\beta}+h x\right) d A(x) \\
\quad=\iint \rho\left(a+t \tau_{\beta}+n \nu_{\beta}\right)\left|c-c_{L_{\beta}}\right|\left(X_{\beta}+h\left(t \tau_{\beta}+n \nu_{\beta}\right)\right) d n d t
\end{aligned}
$$

in terms of the (scaled) tangential and normal variables $(t, n)$. In effect, this final integration is over the intersection of the support $S_{1}$ of $\rho(a+\cdot)$ with the support $S_{2}$ of $\left|c-c_{L_{\beta}}\right|\left(X_{\beta}+h(\cdot)\right)$ ( $\equiv 1$ on $\left.S_{2}\right)$. We are assuming that $\left\|X_{i}-X_{\beta}\right\|=O(h)$.

Suppose first that the support of $\rho$ is bounded. Then, $S_{1}$ is bounded. $S_{2}$ is the set of $(t, n)$ such that $X_{\beta}+h\left(t \tau_{\beta}+n \nu_{\beta}\right)$ lies between $\beta$ and its tangent line $L_{\beta}$. Consequently, the magnitude of the nonzero limit on the inner integral is an $n_{0}(t)$ such that $n_{0} h=(h t)^{2}\left(\left|\kappa_{\beta}\right| / 2+O(1)\right)$, with $\kappa_{\beta}$ the curvature of $\beta$ at $X_{\beta}$ and with $t$ bounded. So, $n_{0}$ and, hence, the integral are $O(h)$ in size. This proves the first half of

Remark 8.1. (The average color near a curved border as a perturbation of the average color of a nearby tangent half-space.) Let $X_{\beta, i}$ be the closest point on $\beta$ to $X_{i}$-at which $X_{\beta, i}-X_{i}$ is orthogonal to the tangent line $L_{\beta, i}$ to $\beta$ at $X_{\beta, i}$ (with normal $\nu_{\beta, i}$ pointing into $\Omega_{1}$ ), and suppose $\beta$ 's curvature is bounded. Then

$$
\bar{c}\left(X_{i}\right)=\bar{c}_{L_{\beta, i}}\left(X_{i}\right)+O(h) .
$$

But, suppose $\beta$ 's curvature is differentiable. Then, with $C_{\beta, i}$ the osculating circular disc to $\beta$ at $X_{\beta, i}$, with $\bar{c}_{C_{\beta, i}}$ the average color function associated with coloring $C_{\beta, i}$ the color appropriate to $\nu_{\beta, i}$, and with $f_{\nu}$ the cumulative distribution function for $\nu$-normal lines (4.2),

$$
\bar{c}\left(X_{i}\right)=\bar{c}_{C_{\beta, i}}\left(X_{i}\right)+O\left(h^{2}\right)=f_{\nu_{\beta, i}}\left(\sigma_{\beta, i}\right)-\kappa_{\beta, i} M_{2, i} h / 2+O\left(h^{2}\right),
$$

where the sign of $\kappa_{\beta, i}$ is taken to be positive if $\beta$ curves toward $\Omega_{1}$ at $X_{\beta, i}$, where $\sigma_{\beta, i}:=\nu_{\beta, i} \cdot\left(X_{i}-X_{\beta, i}\right) / h\left(= \pm\left\|X_{i}-X_{\beta, i}\right\| / h\right)$, and where $M_{2, i}$ is the second moment - of the line with linear density $\rho$-with normal $\nu_{\beta, i}$ through and about the present analog $P_{\sigma_{\beta, i}, \nu_{\beta, i}}$ of the point (8.8).

Proof. In (8.9), now, replace $\beta$ with $\beta, i$ and then $L_{\beta, i}$ with $C_{\beta, i}$. The size of the difference between the limits on the inner integral becomes $n_{1}(t)$ such that $n_{1} h$ is proportional to $(h t)^{3}$.

The estimate $\left(\bar{c}-\bar{c}_{L_{\beta}}\right)\left(X_{i}\right)=O(h)$ can hold even when the support of $\rho$ is the entire plane. For example, suppose $\rho$ is an azimuthally symmetric Gaussian. Then the final integral is bounded by the sum of two integrals-the first from $r_{h}$ to $\infty$ 
with $r_{h}^{2}$ of order $\log \left(1 / h^{2}\right)$ in size; and the second, from 0 to $r_{h}$. In the integrand of the first, bound the absolute value by one and cover all angles; the result is $O\left(h^{2}\right)$ in size. For the second: the $O\left(h t^{2}\right)$ bound on the nontrivial limit for the inner integral holds since $h t=O\left(h r_{h}\right)$ goes to zero; and one concludes the second integral is $O(h)$ in size.

So, to ensure convergence estimable via Proposition 8.1 to the tangent line $L_{\beta}$ for $\beta$ not a line, we will want the $\bar{c}\left(X_{i}\right)$, as perturbations $\tilde{c}_{i}$ there of $\bar{c}_{L_{\beta}}\left(X_{i}\right)$, to be uniformly usable. This motivates

Definition 8.2. The numbers $\bar{c}\left(X_{i}\right)$ (and points $X_{i}$ ) are "usefully usable" means that $O(h)$ perturbations of the numbers $\bar{c}\left(X_{i}\right)$ are uniformly usable (Definition 8.1); more precisely, for each $K>0$ there exists $h_{0}>0$ such that the numbers $\bar{c}\left(X_{i}\right) \pm K h$ are uniformly usable for $h \leq h_{0}$.

Thus, while "usable" implied "uniformly usable" for (6.1), it does not imply "usefully usable". In view of the restriction placed on $\rho$ at the end of Section 4, being between fixed limits $\varepsilon(>0)$ and $1-\varepsilon$ suffices for useful usability.

The following observation will be needed but seems evident.

Remark 8.2. (Line-approximations to curves, in terms of the points and normals that define them.) Suppose (1) the plane curve $\beta$ has bounded curvature; (2) the point $Y$ approximates $\beta$, i.e., there is some $X_{\beta}(Y)$ in $\beta$ so that

$$
\left\|Y-X_{\beta}(Y)\right\|=O\left(\varepsilon_{p}\right)=o(1) \quad(p \text { for "point" }) ;
$$

and (3) the unit vector $\nu$ approximates the unit normal $\nu_{\beta}$ to $\beta$ at $X_{\beta}(Y)$ :

$$
\left\|\nu-\nu_{\beta}\right\|=O\left(\varepsilon_{\nu}\right)=o(1) .
$$

Then, in any neighborhood $N$ of $Y$, of diameter $d=o(1)$, the line $L$ through $Y$ perpendicular to $\nu$ satisfies

$$
\sup _{x \text { in } L \cap N}\|x-\beta\|=O\left(\varepsilon_{p}+d \varepsilon_{\nu}+d^{2}\right) .
$$

If there is a second point $Z$ on $L$ with $\left\|Z-X_{\beta}(Z)\right\|=O\left(\varepsilon_{p}\right)$, then

$$
\left\|\nu-\nu_{\beta}\right\|=O\left(\tilde{\varepsilon}_{\nu}\right), \quad \tilde{\varepsilon}_{\nu}:=\min \left(\varepsilon_{\nu},\|Y-Z\|+\varepsilon_{p} /\|Y-Z\|\right) ;
$$

and, in any neighborhood $N$ of $\overline{Y Z}$, of diameter $d=o(1)$,

$$
\sup _{x \text { in } L \cap N}\|x-\beta\|=O\left(\varepsilon_{p}+d \tilde{\varepsilon}_{\nu}+d^{2}\right) \text {. }
$$

With this, and taking $\varepsilon=O(h)$ in Proposition 8.1 (as we may by Remark 8.1), we have

Proposition 8.2. (Local convergence of Algorithm 7.1's line $L_{A}$ to a border $\beta$ having bounded curvature.) Suppose, in the context of this section's first paragraph, that (1) $\rho$ (4.1) has bounded support or is an azimuthally symmetric Gaussian; (2) the data $\bar{c}\left(X_{i}\right)$ are usefully usable, $i=1,2$; and (3) if $f_{\nu}$ is independent of $\nu$, then $\overline{X_{1} X_{2}}$ is not nearly perpendicular to $\beta$ locally $(\sin \alpha \neq o(1))$, or-in general(8.4) holds. Then $L_{A}$ exists for $\delta$ (already assumed $\left.O(h)\right) \neq O\left(h^{2}\right)$; and with $\Delta_{i}$ (respectively, $\Delta_{\beta, i}$ ) the distance from $X_{i}$ to $L_{A}$ (respectively, $L_{\beta}$ ),

$$
\left\|\nu-\nu_{\beta}\right\|=O\left(h^{2} / \delta\right) ; \quad \text { and } \quad\left|\Delta_{i}-\Delta_{\beta, i}\right|=O\left(h^{3} / \delta\right) \quad\left(\text { resp. } O\left(h^{2}\right)\right),
$$

$$
i=1,2
$$


when $f_{\nu}$ is $\nu$-(in)dependent. Consequently, the two points of tangency of $L_{A}$ to its determining circles, namely,

$$
Y:=X_{1}-h \sigma_{1} \nu, \quad Z:=X_{2}-h \sigma_{2} \nu, \quad \sigma_{i}:=f_{\nu}^{-1}\left(\bar{c}_{i}\right), \quad i=1,2,
$$

satisfy $\left\|Y-X_{\beta, 1}\right\|=O\left(h^{3} / \delta\right)=\left\|Z-X_{\beta, 2}\right\|$ (respectively, $\left.O\left(h^{2}\right)\right)$, with $X_{\beta, i}$ the point on $\beta$ nearest to $X_{i}$ and when $f_{\nu}$ is $\nu$-(in) dependent. So $L_{A}$ is within $O\left(h^{3} / \delta\right)$ of the border $\beta$ throughout any $O(h)$ neighborhood of $\overline{Y Z}$ - and $O\left(h^{2}\right)$ throughout any $O(\delta)$ neighborhood if $f_{\nu}$ is $\nu$-independent.

To complete the analogs of the one-dimensional results mentioned at the end of Section 1: Similar convergence holds for other $O(h)$ perturbations in the $\bar{c}\left(X_{i}\right)$ part of the data $\left(\bar{c}_{i}, X_{i}\right)$-such as would occur if the color $c(x)$ being averaged were nonconstant in the two regions and were known with $O(h)$ accuracy on the support of $\rho\left(\left(\cdot-\overline{X_{1} X_{2}}\right) / h\right)$, and with the jump at the discontinuity not being $o(1)$. The consequences of perturbations in the other part of the data, namely, the $X_{i}$, are similar if $\left\|\tilde{X}_{i}-X_{i}\right\|=O(h \delta), i=1,2$.

If $f_{\nu}$ is $\nu$-independent but $\overline{X_{1} X_{2}}$ can become perpendicular to $\beta$ (e.g., were $\sin \alpha=O(h))$, and if $h$ and $\delta$ are of comparable size, then, according to Remark 8.1 and Proposition 8.1, the local accuracies of $\nu$ and of $L_{A}$ (when they exist) are a priori $O\left(h^{1 / 2}\right)$ and $O\left(h^{3 / 2}\right)$, respectively - and these estimates will then be sharp unless $\beta$ 's curvature becomes small or the two relevant specific second moments for $\nu$-normal lines become (fortuitously) close (cf. (9.3) ff. below). We also conclude from Remark 8.2 and (8.11) the a posteriori estimate that $L_{A}$ is $O\left(h^{2}+\|Y-Z\|^{2}\right)$ accurate throughout any $O(\|Y-Z\|)$ neighborhood of $\overline{Y Z}$. We have nothing useful to conclude if $f_{\nu}$ is $\nu$-dependent but (8.4) fails.

9. Curvature Estimates: Problems, and a Solution. Another characteristic of a border one might want to recover from discrete values of its blurring is its curvature; see, e.g., Chorin [8] for the blurring (1.2).

Our border approximations, applied in a sequence of overlapping neighborhoods of diameter $O(h)$, could yield a broken line which is (usually) within order $O\left(h^{3} / \delta\right)$ of the border $\beta$. More to the point: with each data pair $\left(X_{j}, \bar{c}_{j}\right),\left(X_{j+1}, \bar{c}_{j+1}\right)$ we have associated a line segment - the one connecting the two points of tangency $Y_{j+1 / 2}, Z_{j+1 / 2}(8.12)-$ which locally approximates $\beta$. It is natural to associate this segment's unit normal

$$
\nu=: \nu_{j+1 / 2} \quad \text { with } \beta \text { 's normal near } \quad \bar{X}_{j+1 / 2}:=\left(Y_{j+1 / 2}+Z_{j+1 / 2}\right) / 2
$$

(more precisely, halfway between $X_{\beta, j}$ and $X_{\beta, j+1}$ on $\beta$ ); and this prompts an estimate such as

$$
\begin{aligned}
& \left|\bar{\kappa}_{j}\right|:=\left|\nu_{j-1 / 2} \times \nu_{j+1 / 2}\right| /\left\|\bar{X}_{j+1 / 2}-\bar{X}_{j-1 / 2}\right\|, \text { in which } U \times V:= \\
& u_{1} v_{2}-u_{2} v_{1} \text { is the only nontrivial component of the usual vector } \\
& \text { product of } U \text { and } V \text { appropriately embedded in right-handed } 3- \\
& \text { space, }
\end{aligned}
$$

for the size $\left\|\left(d^{2} X / d s^{2}\right)(s)\right\|=:|\kappa(s)|$ of the curvature of $\beta=X(s), s$ being arclength, near the two points $X_{\beta, j-1}$ and $X_{\beta, j+1}$ on $\beta$ (and analogous to Mjolsness and Swartz $[21])$. 
Unfortunately, even for smooth curvatures, this (and similar) elementary estimates do not converge in general unless the curvature is zero at $\lim _{h \rightarrow 0} X_{\beta, j}$. We sketch the reason. Like for the vector product of the normals of two adjacent secant segments, the unit vector $\nu_{j+1 / 2}$-regarded as approximating $\beta$ 's normal $\nu_{\beta}$ halfway between $X_{\beta, j}$ and $X_{\beta, j+1}$-is convergent, so its principal error vector is essentially parallel to $\beta$. Also, the size of this error is proportional to the scale $h$ and to the smoothly varying local curvature $\kappa_{\beta, j+1 / 2}$. However, unlike for the secants' normals, the error also involves a geometric factor, neither smoothly varying nor generally $o(1)$, depending not only on the orientation of the two points $X_{j}, X_{j+1}$ with respect to $\beta$ but also on their distances (relative to the scale $h$ ) from $\beta$ (see (9.6)). Hence, only if the relative geometry of the tangent line and the three points is fixed on the $h$ scale, will the numbers (9.2) converge-and, then, to a fixed multiple of the local curvature. Only when this multiple is one-so for almost no relative geometries - has one the convergence desired. The remedy is clear: incorporate sufficiently accurate estimates of the geometric factor into the curvature estimate (9.2), thereby allowing the higher-order error terms to dominate. These observations - and, so, the remedy - are the content of remarks culminating in Proposition 9.2.

Remark 9.1. Let $v:=(\cos \alpha, \sin \alpha)$ and $u:=(\sin \alpha,-\cos \alpha)$ so that $(u, v)$ constitutes a basis for a right-handed coordinate system for the plane. Suppose $\cos \tilde{\alpha}=\cos \alpha+\varepsilon$ with $\varepsilon=o\left(\sin ^{3 / 2} \alpha\right)$, and define $\tilde{v}$ analogously. Then

$$
\tilde{v}=v+(\varepsilon / \sin \alpha) u+O\left(\varepsilon^{2} / \sin ^{3} \alpha\right)
$$

Proof. Multiply

$$
|\sin \tilde{\alpha}|=\sqrt{1-(\cos \alpha+\varepsilon)^{2}}=|\sin \alpha|-(\varepsilon /|\sin \alpha|)(\cos \alpha-\varepsilon / 2)+O\left(\varepsilon^{2} / \sin ^{3} \alpha\right)
$$

by $\operatorname{sgn}(\sin \tilde{\alpha})=\operatorname{sgn}(\sin \alpha)$ for $\varepsilon$ sufficiently small.

We now consider the error in $\nu_{j+1 / 2}$ (7.2) as an estimate of $\nu_{\beta, j+1 / 2}$, i.e., halfway between $X_{\beta, j}$ and $X_{\beta, j+1}$ on $\beta$. We assume $\kappa$ is differentiable. Applying $f_{\nu_{j+1 / 2}}^{-1}$ to $(8.10)$, and since $\left(f_{\nu_{j+1 / 2}}^{-1}\right)^{\prime}\left(f_{\nu_{j+1 / 2}}\left(\sigma_{\beta, j}\right)\right)=1 /\left(d f_{\nu_{j+1 / 2}} / d \sigma\right)\left(\sigma_{\beta, j}\right)$ with $\sigma_{\beta, j}=$ $\sigma_{j}+O(h)$ (and with $f_{\nu_{j+1 / 2}}\left(\sigma_{j}\right)=\bar{c}\left(X_{j}\right)$ ), we conclude more precisely that

$$
f_{\nu_{j+1 / 2}}^{-1}\left(\bar{c}\left(X_{j}\right)\right)=\sigma_{\beta, j}-\kappa_{\beta, j} \mu_{j}^{(2)} h(1 / 2+O(h)), \quad \text { with } \quad \mu_{j}^{(2)}:=M_{2, j} / M_{0, j}
$$

the specific second moment for $\nu$-normal lines, i.e., the ratio of the second moment to the zeroth moment (this last being $f_{\nu_{j+1 / 2}}^{\prime}$ ), of the line segment with linear density $\rho$ and normal $\nu_{j+1 / 2}=\nu_{\beta, j+1 / 2}+O\left(h^{2} / \delta_{j+1 / 2}\right)$, through and about the point $P_{\sigma_{j}, \nu_{j+1 / 2}}$ (8.8). From (7.2) and (9.3), then, and as $\kappa_{\beta, j}=\kappa_{\beta, j+1 / 2}\left(1+O\left(\delta_{j+1 / 2}\right)\right)$ with $\kappa_{\beta, j+1 / 2}$ the curvature halfway between $X_{\beta, j}$ and $X_{\beta, j+1}$, and with $\delta_{j+1 / 2}=$ $\left\|X_{j+1}-X_{j}\right\|$,

$$
\begin{aligned}
\cos L_{\tau_{j+1 / 2}, \nu_{j+1 / 2}}= & \left(\sigma_{\beta, j+1}-\sigma_{\beta, j}\right) h / \delta_{j+1 / 2} \\
& -\kappa_{\beta, j+1 / 2}\left(\mu_{j+1}^{(2)}-\mu_{j}^{(2)}\right)\left(h^{2} / \delta_{j+1 / 2}\right)(1 / 2+O(h)) .
\end{aligned}
$$

But, in Appendix 3, we show that, if $\nu_{\beta, j+1 / 2}$ is $\beta$ 's unit normal "halfway between" $\nu_{\beta, j}$ and $\nu_{\beta, j+1}$, then

$$
\left(\sigma_{\beta, j+1}-\sigma_{\beta, j}\right) h / \delta_{j+1 / 2}=\cos L_{\tau_{j+1 / 2}, \nu_{\beta, j+1 / 2}}\left(1+O\left(\kappa_{\beta, j+1 / 2}^{2} \delta_{j+1 / 2}^{2}\right)\right)
$$


for geometrical reasons, i.e., independent of $\rho$. With this, we have proved

Remark 9.2. Suppose $\beta$ 's curvature is differentiable. Let

$$
\theta_{j+1 / 2}:=\left(\mu_{j+1}^{(2)}-\mu_{j}^{(2)}\right) / 2
$$

be half the difference of the two appropriate specific second moments in (9.3). Then

$$
\cos L_{\tau_{j+1 / 2}, \nu_{\beta, j+1 / 2}}=\cos L_{\tau_{j+1 / 2}, \nu_{j+1 / 2}}+\varepsilon_{j+1 / 2}+O\left(h^{3} / \delta_{j+1 / 2}+\delta_{j+1 / 2}^{2}\right),
$$

where $\varepsilon_{j+1 / 2}:=\kappa_{\beta, j+1 / 2} \theta_{j+1 / 2}\left(h^{2} / \delta_{j+1 / 2}\right)$.

Thus, if we knew the curvature locally, we could better estimate $\nu$ locally:

Remark 9.3. Pick $t_{j+1 / 2}$ so that $\left(t_{j+1 / 2}, \nu_{j+1 / 2}\right)$ is a right-handed orthonormal basis. If $\tilde{\nu}_{j+1 / 2}$ is given by

$$
\tilde{\nu}_{j+1 / 2}:=\nu_{j+1 / 2}+\kappa_{\beta, j+1 / 2} \theta_{j+1 / 2} h^{2} t_{j+1 / 2} / \Delta X_{j+1 / 2},
$$

where $\Delta X_{j+1 / 2}:=\delta_{j+1 / 2} \sin L_{\tau_{j+1 / 2}, \nu_{j+1 / 2}}$ is the length of the line segment of tangency $\overline{Y_{j+1 / 2} Z_{j+1 / 2}}$ (and presuming the sine nonnegative), then (compare (8.11))

$$
\tilde{\nu}_{j+1 / 2}=\nu_{\beta, j+1 / 2}+O\left(h^{3} / \delta_{j+1 / 2}\right)
$$

Proof. Combine Remarks 9.1 and 9.2.

As an aside, we conclude

PROPOSITION 9.1. (Improved error estimates for Algorithm 7.1.) Under the assumptions of Proposition 8.2-except that the border's curvature $\kappa$ is now presumed differentiable and first-order $\sigma$-difference quotients of the specific second moment $\mu^{(2)}(\sigma, \nu)$ (9.3) are now presumed uniformly bounded ${ }^{\dagger}$ over relevant triples $\left(\sigma_{1}, \sigma_{2}, \nu\right)$-one has $\left|\Delta_{i}-\Delta_{\beta, i}\right|=O\left(h^{2}\right), i=1,2$, and

$$
\begin{aligned}
\left\|\nu-\nu_{\beta, 3 / 2}\right\|= & O(h) \\
& \left(\neq o(h) \text { unless } \kappa_{\beta, 3 / 2}=o(1) \text { or } \overline{X_{1} X_{2}} \text { becomes parallel to } \beta\right) .
\end{aligned}
$$

Consequently, the two points of tangency of $L_{A}$ to its determining circles, namely,

$$
Y:=X_{1}-h \sigma_{1} \nu, \quad Z:=X_{2}-h \sigma_{2} \nu, \quad \sigma_{i}:=f_{\nu}^{-1}\left(\bar{c}_{i}\right), \quad i=1,2,
$$

\footnotetext{
${ }^{\dagger}$ This condition is not unduly restrictive. For example, suppose the density $\rho$ (4.1) were constant on its support $S$; and suppose $S$ were convex with a continuous, piecewise smooth boundary $\partial S$. Let $(\eta, \sigma)$ be the coordinates of an orthogonal coordinate system based on $(t, \nu)$. Then $\partial S$ defines two continuous, piecewise smooth functions $\eta_{ \pm}(\sigma)$ on an open interval $I_{\nu}$ of the $\sigma$-axis; and the difference quotient of each is bounded with one possible exception, namely, when both $\sigma_{i}$ approach the same end of $I_{\nu}$. Extended by continuity to $I_{\nu}$ 's closure, the graphs of $\eta_{+}$ and $\eta_{-}$may connect (their union then reproducing $\partial S$ ), or they may not connect (should $\partial S$ contain a line segment perpendicular to $\nu$ ). In any case, the difference quotient $D$ of $\mu^{(2)}$ is a fixed multiple of the difference quotient of the function $\eta_{+}^{2}+\eta_{+} \eta_{-}+\eta_{-}^{2}$. Hence, conditions sufficient for bounded $D$ include: (a) the curvature of $\partial S$ (defined piecewise and already presumed of one sign) is bounded away from zero (invoke (10.2) for this should both $\sigma_{i}$ get close to the same end of $I_{\nu}$ ), or (b) at each point of $\partial S$ having zero left or right curvatures (excepting the interior of line segments) the tangent is discontinuous, or (c) the two $\sigma_{i}$ do not simultaneously approach the same end of $I_{\nu}$ (possibly already a consequence of the "useful usability" assumption on the data). For azimuthally symmetric (but not necessarily constant) densities $\rho$, see Section 10-in particular, condition (c) suffices for the density (6.3).
} 
satisfy $\left\|Y-X_{\beta, 1}\right\|=O\left(h^{2}\right)=\left\|Z-X_{\beta, 2}\right\|$, with $X_{\beta, i}$ the point on $\beta$ nearest to $X_{i}$. So $L_{A}$ is within $O\left(h^{2}\right)$ of the border $\beta$ throughout any $O(h)$ neighborhood of $\overline{Y Z}$.

Proof. Use $j=1, \delta_{3 / 2}=\delta=\left\|X_{2}-X_{1}\right\|$ and similarly for $\tau$ and $\nu$, and (7.2) in the form

$$
h / \delta=\left(\cos L_{\tau, \nu}\right) /\left(\sigma_{2}-\sigma_{1}\right)
$$

to conclude from Remark 9.3 that

$$
2\left\|\nu-\tilde{\nu}_{3 / 2}\right\|=h\left|\kappa_{\beta, 3 / 2}\left(\cot L_{\tau, \nu}\right)\left(\mu^{(2)}\left(\sigma_{2}, \nu\right)-\mu^{(2)}\left(\sigma_{1}, \nu\right)\right) /\left(\sigma_{2}-\sigma_{1}\right)\right|,
$$

with $\mu^{(2)}$ the second specific moment (9.3) about $P_{\sigma, \nu}$ (8.8). Then apply (9.5), noting $\delta \neq O\left(h^{2}\right)$.

More importantly, we can successfully adapt various forms of curvature estimates which were originally designed to use the (exact) locations of three adjacent points on a plane curve. Such adaptation must bear three points in mind: (1) Orientation: $\beta$ 's unit normal $\nu_{\beta}$ points into $\Omega_{1}$, so the appropriate unit tangent $t_{\beta}=d X(s) / d s$ is parallel to $\beta$ with $\Omega_{1}$ to the left side of $t_{\beta}$, and this means that the direction arclength $s$ increases on $\beta$ is predetermined. (2) We shall be invoking Remark 9.3 to obtain convergent curvature estimates, so the sign of the curvature is also crucial - and we have chosen it (below (8.10)) to be positive if $\beta$ curves towards $\Omega_{1}$. Thus, the (signed) curvature we shall approximate is

$$
\kappa_{\beta}:=\nu_{\beta} \cdot d t_{\beta} / d s=-t_{\beta} \cdot d \nu_{\beta} / d s .
$$

(3) The line segments $\overline{Y_{j+1 / 2} Z_{j+1 / 2}}$ approximating $\beta$-even the pair associated with two "adjacent" data-pairs $X_{i-1}, X_{i}$ and $X_{i}, X_{i+1}$-need contain no common point and, if they do, it will not be an endpoint in general. Hence these segments will have to play a secondary role - not unreasonable in view of (9.5) - unless joined up somehow to form continuous broken lines approximating $\beta$.

Of the four types of three-point curvature estimates (see, e.g., Mjolsness and Swartz [21]), we choose to adapt one--there called $\kappa_{\times}$-as follows:

PROPOSITION 9.2. (A convergent local curvature estimate.) From the four usefully usable average colors (Definition 8.2) associated with two pairs of points $X_{0}, X_{1}$ and $X_{2}, X_{3}$-and supposing the conditions of Proposition 9.1 hold for each pair-determine normals $\nu_{1 / 2}, \nu_{5 / 2}$ and associated points $\bar{X}_{1 / 2}, \bar{X}_{5 / 2}$ midway between the corresponding two points $Y_{j+1 / 2}, Z_{j+1 / 2}$ of tangency, $j=0,2$ (Proposition 8.2). We assume $\overline{X_{0} X_{1}} \neq \bar{X}_{2} \bar{X}_{3}$. We also presume (without loss) that the four points are oriented as follows. (1) Points within pairs: for $j=0,2$, with the basis $\left(t_{j+1 / 2}, \nu_{j+1 / 2}\right)$ right-handed as in Remark 9.3 , and with $\tau_{j+1 / 2}$ the unit vector from $X_{j}$ to $X_{j+1}$, we presume $\tau_{j+1 / 2} \cdot t_{j+1 / 2}>\varepsilon>0$ uniformly (otherwise, relabel $X_{j}$ and $\left.X_{j+1}\right)$. (2) Then the two pairs: we presume $\left(t_{1 / 2}+t_{5 / 2}\right) \cdot\left(\bar{X}_{5 / 2}-\bar{X}_{1 / 2}\right) \geq$ 0 (otherwise, relabel). Define

$$
\begin{aligned}
\Delta s & :=\left\|\bar{X}_{5 / 2}-\bar{X}_{1 / 2}\right\| \quad \text { and } \\
\delta_{j+1 / 2}: & =\left\|X_{j+1}-X_{j}\right\|, \quad \Delta X_{j+1 / 2}:=\left\|Z_{j+1 / 2}-Y_{j+1 / 2}\right\|, \quad j=0,2 .
\end{aligned}
$$

Use $\sigma_{0}, \sigma_{1}$, and $\nu_{1 / 2}$ to compute from $\rho$ the specific second moments $\mu_{0}^{(2)}$ and $\mu_{1}^{(2)}$ (9.3) about the two points $P_{\sigma_{j}, \nu_{1 / 2}}(8.8)$; and $\sigma_{2}, \sigma_{3}$, and $\nu_{5 / 2}$ to compute $\mu_{2}^{(2)}$ and 
$\mu_{3}^{(2)}$. Define

$$
M:=\left(\left(\mu_{3}^{(2)}-\mu_{2}^{(2)}\right) / \Delta X_{5 / 2}-\left(\mu_{1}^{(2)}-\mu_{0}^{(2)}\right) / \Delta X_{1 / 2}\right)\left(h^{2} /(2 \Delta s)\right)
$$

(see also (10.3)-(10.5)). In general, $M \neq o(1)$. Assume, finally, that $1+M \neq o(1)$ and $\min \left(\delta_{1 / 2}, \delta_{5 / 2}\right) \neq O\left(h^{2}\right)$. Then the approximate (signed) curvature

$$
\hat{\kappa}:=\left(\nu_{1 / 2} \times \nu_{5 / 2}\right) /((1+M) \Delta s) \quad(\times \text { as in }(9.2))
$$

admits the error bound $\hat{\kappa}=\hat{\kappa}_{\beta}+O(E)$, with

$$
E:=\Delta s+h\left(\frac{h}{\Delta s}+\left(1+\frac{h}{\Delta s}\right) \frac{h}{\min \left(\delta_{1 / 2}, \delta_{5 / 2}\right)}\right),
$$

and with $\hat{\kappa}_{\beta}$ being $\beta$ 's curvature at a point in the smallest interval (on $\beta$ ) containing the four points $X_{\beta, i}$ (on $\beta$ ) closest, respectively, to $X_{i}, i=0, \ldots, 3$. In particular, if $\Delta s$ and $\min \left(\delta_{1 / 2}, \delta_{5 / 2}\right)$ are both of exact order $h$ and if $1+M \neq o(1)$, then $E=O(h)$.

Proof. It follows - first from (9.6) and elementary approximation results, and second from (9.5) and Proposition 8.2-that, with $\Delta s_{\beta}:=\left\|X_{\beta, 5 / 2}-X_{\beta, 1 / 2}\right\|$, and with $\hat{\kappa}_{\beta}$ the curvature halfway between $X_{\beta, 1 / 2}$ and $X_{\beta, 5 / 2}$ on $\beta$,

$$
\begin{aligned}
& \hat{\kappa}_{\beta}=\left(\nu_{\beta, 1 / 2} \times \nu_{\beta, 5 / 2}\right) / \Delta s_{\beta}+O\left(\Delta s_{\beta}\right)=\left(\tilde{\nu}_{1 / 2} \times \tilde{\nu}_{5 / 2}\right) / \Delta s+E_{1}, \\
& E_{1}=O\left(\Delta s+h^{2} / \Delta s+h^{3} /\left(\Delta s \min \left(\delta_{1 / 2}, \delta_{5 / 2}\right)\right) .\right.
\end{aligned}
$$

From this, (9.8)-(9.9), Remark 9.3 (since $\varepsilon<L_{\tau_{j+1 / 2}, \nu_{j+1 / 2}}<\pi-\varepsilon$ ), the properties of the vector product, and Proposition 8.2,

$$
\begin{aligned}
& \hat{\kappa}_{\beta}=\left(\nu_{1 / 2} \times \nu_{5 / 2}\right) / \Delta s-M\left(1+E_{2}\right) \hat{\kappa}_{\beta}+E_{1}+E_{2}^{2}, \\
& E_{2}=O\left(h^{2} / \min \left(\Delta X_{1 / 2}, \Delta X_{5 / 2}\right)\right)=O\left(h^{2} / \min \left(\delta_{1 / 2}, \delta_{5 / 2}\right)\right) .
\end{aligned}
$$

$M$ is not $o(1)$ in general (see, e.g., (10.3)-(10.5)), but $1+M \neq o(1)$ by assumption. Hence, from (9.10)-(9.12),

$$
\left|\hat{\kappa}-\hat{\kappa}_{\beta}\right|=\left|E_{1}+E_{2}^{2}-M E_{2}\right| /|1+M|=O\left(E_{1}+E_{2}\right),
$$

and the error bound follows.

Remark 9.4 . One could equally well replace $\Delta s(9.8)$ with the distance from $\bar{X}_{1 / 2}$ to $\bar{X}_{5 / 2}$ as measured along the single-jointed, continuous broken line containing $\overline{Y_{1 / 2} Z_{1 / 2}}$ and $\overline{Y_{5 / 2} Z_{5 / 2}}$.

This larger but more complicated $\Delta s$ would keep $\hat{\kappa}$ under better control. Moreover, if all four $\sigma_{j}$ were now zero (so that the two $\mu^{(2)}$-differences in $M$, and hence $M$, were zero), and if $X_{1}=X_{2}$, then $|\hat{\kappa}|$ would coincide with $\left|\kappa_{\times}\right|$(of Mjolsness and Swartz [21]) based on $X_{0}, X_{1}$, and $X_{3}$.

There are other approaches to curvature estimation which retain the general viewpoint of this paper. For example: it would be interesting if one of the (up to eight) circles mutually tangent to three neighboring small circles (7.3) proved to have its curvature locally convergent.

10. Approximate Curvature Using Disc-Averaged Colors. When the density $\rho$ (4.1) used for scaled color-averaging is azimuthally symmetric about the origin, the specific second moment $\mu^{(2)}(9.3)$, as well as the cumulative distribution function $f_{\nu}$ for $\nu$-normal lines, is independent of $\nu$ and so depends only on $\sigma$. In 
particular, the specific second moment for a probability distribution $\rho(r), r:=$ $\sqrt{x^{2}+y^{2}}$, on the disc of diameter 1 , is, for $0 \leq|\sigma|<1 / 2$,

$$
\mu^{(2)}(\sigma)=\int_{0}^{\sqrt{1 / 4-\sigma^{2}}} z^{2} \rho\left(\sqrt{\sigma^{2}+z^{2}}\right) d z / \int_{0}^{\sqrt{1 / 4-\sigma^{2}}} \rho\left(\sqrt{\sigma^{2}+z^{2}}\right) d z .
$$

For example, the specific second moments for the densities associated with Sections 5 and 6 , respectively, are

$$
\mu_{\mathrm{V}}^{(2)}(\sigma):=\left(1 / 4-\sigma^{2}\right) / 3 \text { and } \mu_{\mathrm{VI}}^{(2)}(\sigma):=\left(1 / 4-\sigma^{2}\right)^{1 / 2} / 4
$$

To promote further analysis, we now relate $M(9.9)$ to a difference approximation to the second derivative of a scalar function $y(x)$ of one variable $x$. This particular approximation, a difference quotient of the difference of the slope of two secant lines, is expressed in terms of four values $x_{0}, \ldots, x_{3}$ and corresponding values $y_{i}:=y\left(x_{i}\right)$ as

$$
\begin{aligned}
\left(\mathscr{D}^{2} y\right)_{3 / 2} & :=\left((\mathscr{D} y)_{5 / 2}-(\mathscr{D} y)_{1 / 2}\right) /\left(\bar{x}_{5 / 2}-\bar{x}_{1 / 2}\right), \quad \text { in which } \\
(\mathscr{D} y)_{j+1 / 2} & :=\left(y_{j+1}-y_{j}\right) /\left(x_{j+1}-x_{j}\right), \quad \bar{x}_{j+1 / 2}:=\left(x_{j+1}+x_{j}\right) / 2
\end{aligned}
$$

and it yields the (constant) second derivative of quadratic functions $y(x)$ while reducing to the ordinary second difference quotient if $x_{1}=x_{2}$ and $y_{1}=y_{2}$. Now, under the assumptions of Proposition 9.2, from (7.2), and for $j=0,2$,

$$
\begin{aligned}
\Delta X_{j+1 / 2} & =\delta_{j+1 / 2} \sin \alpha_{j+1 / 2} \text { and } \sigma_{j+1}-\sigma_{j}=\left(\delta_{j+1 / 2} / h\right) \cos \alpha_{j+1 / 2} \\
\alpha_{j+1 / 2} & :=L_{\tau_{j+1 / 2}, \nu_{j+1 / 2}}
\end{aligned}
$$

being in $[\varepsilon, \pi-\varepsilon]$. Consequently, $M(9.9)$ is also given by

$$
M=(h / 2)\left(\left(\mathscr{D} \mu^{(2)}\right)_{5 / 2} \cot \alpha_{5 / 2}-\left(\mathscr{D} \mu^{(2)}\right)_{1 / 2} \cot \alpha_{1 / 2}\right) / \Delta s .
$$

In particular, it works out that,

when the segments $\overline{X_{0} X_{1}}$ and $\overline{X_{2} X_{3}}$ lie in the same line, $M=$ $\left(\mathscr{D}^{2} \mu^{(2)}\right)_{3 / 2}\left(\cot \alpha_{1 / 2}\right)^{2} / 2+O\left(h^{2} / \min \left(\delta_{1 / 2}, \delta_{5 / 2}\right)\right)=o(1)$ if this line becomes parallel to $\beta$.

The first or second difference quotients required in (10.5) or (10.6) are easily calculated for quadratic functions like $\mu_{\mathrm{V}}^{(2)}(10.2)$-and would be particularly simple for $\mu^{(2)}$ linear:

PROPOSITION 10.1. Let $\rho_{c}(r)$ (c for "curvature") be a probability distribution on the disc of diameter one solving (10.7) below, so that its specific second moment, for $\nu$-normal lines, is piecewise linear as described by

$$
\mu_{c}^{(2)}(\sigma):=a|\sigma|+b, \quad 0 \leq|\sigma| \leq 1 / 2 .
$$

Then, under the hypotheses of Proposition 9.2, with the further restriction if $a \neq 0$ that $\sigma_{0} \sigma_{1} \geq 0$ and $\sigma_{2} \sigma_{3} \geq 0, M(9.9)$ is given by

$$
M=(a h / 2)\left(\operatorname{sgn} \sigma_{2} \cot \alpha_{5 / 2}-\operatorname{sgn} \sigma_{0} \cot \alpha_{1 / 2}\right) / \Delta s,
$$

$\alpha_{j+1 / 2}:=L_{\tau_{j+1 / 2}, \nu_{j+1 / 2}}$. In particular, if $a=0$, or if the signs of $\sigma_{0}, \ldots, \sigma_{3}$ all agree and the segments $\overline{X_{0} X_{1}}$ and $\overline{X_{2} X_{3}}$ lie in the same line, then $M=$ $O\left(h^{2} / \min \left(\delta_{1 / 2}, \delta_{5 / 2}\right)\right)$, so that then

$$
\hat{\kappa}=\left(\nu_{1 / 2} \times \nu_{5 / 2}\right) / \Delta s
$$

itself now converges to the local curvature as described in that proposition. 
Proof. We must solve (10.1) for $\rho=\rho_{c}$, given that $\mu^{(2)}$ there is $\mu_{c}^{(2)}$. This is equivalent to

$$
\gamma(\sigma) \int_{0}^{\sqrt{1 / 4-\sigma^{2}}} \rho_{c}\left(\sqrt{\sigma^{2}+z^{2}}\right) d z=\int_{0}^{\sqrt{1 / 4-\sigma^{2}}}\left(\sigma^{2}+z^{2}\right) \rho_{c}\left(\sqrt{\sigma^{2}+z^{2}}\right) d z
$$

with $\gamma(\sigma):=\mu^{(2)}(\sigma)+\sigma^{2}$. The changes of variables (see, e.g., Faber and Wing [13]), which converted (6.2) to Abel's integral equation, transform (10.7a) into

$$
\begin{aligned}
& \gamma(\sqrt{1 / 4-x}) \int_{0}^{x} \rho_{c}(\sqrt{1 / 4-y}) d y / \sqrt{x-y} \\
& \quad=\int_{0}^{x}(1 / 4-y)^{3 / 2} \rho_{c}(\sqrt{1 / 4-y}) d y / \sqrt{x-y} .
\end{aligned}
$$

Unfortunately, we have failed to solve either of these functional equations and even to show when solutions exist.

11. Border Approximation Using a Widely-Based Approximate Normal. Up to this point we have been intent on abstracting as much knowledge as possible from a minimal amount of data, namely, from the (usefully usable) values of the average color function at two locations. In the next two sections we propose algorithms, yielding lines approximating the border $\beta$ locally (i.e., in neighborhoods of diameter $O(h)$ ), which involve redundant data instead-namely, its usefully usable values at three locations.

The unit normal $\nu$ of a line $L_{\nu}$ must be convergent to the border's local normal if $L_{\nu}$ is to be better than first-order accurate in its local length, and $\nu$ must be first-order accurate if $L_{\nu}$ is second-order accurate locally. One way to insure this, given a usefully usable (Definition 8.2 ) point $Y$, is to let $\hat{\nu}$ be the normal to $\overline{Z_{1} Z_{2}}$ pointing into $\Omega_{1}$, with usefully usable $Z_{1}$ and $Z_{2}$ chosen (a) within $O(\sqrt{h})$ of $Y$, and so that (b) $\left\|Z_{2}-Z_{1}\right\|$ is exactly of order $\sqrt{h}$; for then $\hat{\nu}$ is $O(\sqrt{h})$ accurate locally. Better yet, if such $Z_{1}$ and $Z_{2}$ are chosen so that (c) the projection of $Y$, along $\hat{\nu}$, onto $\overline{Z_{1} Z_{2}}$ is within $O(h)$ of the midpoint of $\overline{Z_{1} Z_{2}}$, then $\hat{\nu}$ can be used to determine a $\nu$ which is locally $O(h)$ accurate. This is the basis for the only algorithm we offer, not requiring iteration when $f_{\nu}$ depends upon $\nu$ :

ALGORITHM 11.1. With $Y, Z_{1}, Z_{2}$, and $\hat{\nu}$ satisfying (a)-(c), and with $f_{\nu}(4.2)$ the cumulative distribution function for $\nu$-normal lines associated with $\rho(4.1)$, let

$$
W_{i}:=Z_{i}-h f_{\hat{\nu}}^{-1}\left(\bar{c}\left(Z_{i}\right)\right) \hat{\nu}, \quad i=1,2 .
$$

With $\nu$ the unit normal to $\overline{W_{1} W_{2}}$ pointing into $\Omega_{1}$, set

$$
X:=Y-h f_{\nu}^{-1}(\bar{c}(Y)) \nu .
$$

The line $L_{A}$ through $X$ perpendicular to $\nu$ is the local approximation to $\beta$.

Remarks 11.1. (1) For $n$ dimensions, suitably restricted densities $\rho$ (Appendix 1), and with all points mentioned being usefully usable: one could find a nondegenerate (cf. Section 12) simplex $\overline{Z_{1} \cdots Z_{n}}$ having its ordinary center of mass appropriately close to $Y$ 's projection along its normal $\hat{\nu}$. Easier to implement would be the use of $n$ uniformly independent line segments $\overline{Z_{1, j} Z_{2, j}}$, the midpoint of each being 
appropriately close to $Y$ 's orthogonal projection on it. (2) As with Algorithm 7.1 (cf. Section 9), precise error estimates for $\nu$ will contain first-order terms involving the local curvature and specific second moments of appropriate line segments. Hence, curvature estimates equivalent to the ordinary use of adjacently-determined normals would again be bounded but unlikely to converge, while an appropriately adapted version of Proposition 9.2 would yield convergent local curvature estimates. (3) Unlike Algorithm 7.1, the linear border produced is not an interpolant -in the sense that it need not yield the data. This allows, for example, the existence of the line $L_{A}$, even when $f_{\nu}$ is independent of $\nu$ but the data varies too rapidly to be yielded by any linear border. (4) The associated operator is not a projector.

PROPOSITION 11.1. (Convergence of Algorithm 11.1.). Suppose the border $\beta$ is thrice differentiable, and that $\rho$ either has compact support or is an azimuthally symmetric Gaussian. Then $X$ lies within $O\left(h^{2}\right)$ of $\beta$, and the line $L_{A}$ lies within $O\left(h^{2}\right)$ of $\beta$ throughout any $O(h)$-size neighborhood of $X$.

Sketch of Proof. Set $\varepsilon:=\sqrt{h}$. In view of $(8.10)$ and the boundedness of $\left(f_{(\cdot)}^{-1}\right)^{\prime}$ on usefully usable data, it follows from (a)-(c) that $\overline{Z_{1} Z_{2}}$ is an $O(h)=O\left(\varepsilon^{2}\right)$ perturbation of $\overline{Z_{1, \beta} Z_{2, \beta}}$, with $Z_{1, \beta}$ (resp., $Z_{2, \beta}$ ) the closest point on $\beta$ to $Z_{1}$ (resp., $Z_{2}$ ), the line segments having length of exact order $\varepsilon$. As the projection of $Y$, along $\hat{\nu}$, onto $\overline{Z_{1, \beta} Z_{2, \beta}}$ is within $O\left(\varepsilon^{2}\right)$ of the midpoints of either segment, and as $\beta$ is thrice differentiable, the normal to $\overline{Z_{1, \beta} Z_{2, \beta}}$ is $O\left(\varepsilon^{2}\right)$ accurate locally, i.e., in an $O\left(\varepsilon^{2}\right)=O(h)$ neighborhood of $Y$. Unfortunately, $\hat{\nu}$ is only $O(\varepsilon)$ accurate locally (unless, fortuitously, $\bar{c}\left(Z_{1}\right)=\bar{c}\left(Z_{2}\right)+o(1)$ ). However, each $W_{i}$ is within $O\left(h^{3 / 2}\right)=O\left(\varepsilon^{3}\right)$ of its corresponding $Z_{i, \beta}$, whence $\nu$ is $O\left(\varepsilon^{2}\right)=O(h)$ accurate locally. These accuracies for the $W_{i}$ and, so, $\nu$-and consequent $O\left(h^{2}\right)$ accuracy for $X$-follow from (8.10), (7.2), and the boundedness of $\left(f_{(\cdot)}^{-1}\right)^{\prime}$ on usefully usable average colors.

It is true that the component of $\hat{\nu}$ normal to $\beta$ is $O(h)$ accurate locally. For any such $\hat{\nu}$, and if $f_{\nu}$ were independent of $\nu$ one could then skip (11.1), use $\hat{\nu}$ for $\nu$ in (11.2), and would find that $X$ would be within $O\left(h^{2}\right)$ of $\beta$. But, one could not then move parallel to $\overline{Z_{1} Z_{2}}$ more than $O\left(h^{3 / 2}\right)$ without losing second-order accuracy. However, one could go on to a usefully usable neighbor $\tilde{Y}$ within $O(h)$ of $Y$, compute the corresponding $\tilde{X}$ (without altering $\hat{\nu}$ ), and the line containing both $X$ and $\tilde{X}$ would be $O\left(h^{2}+\|X-\tilde{X}\|^{2}\right)$ accurate throughout any $O(\|X-\tilde{X}\|)$ neighborhood of $X$ and $\tilde{X}$ (Remark 8.2). But, this seems relatively complicated compared to the application of Algorithm 7.1 under the same conditions - at least, when that algorithm has a solution.

\section{Border Approximation Using a Locally-Based Approximate Nor-} mal. In this section we develop an algorithm involving the usefully usable values of the average color at three adjacent, but strictly noncolinear, locations. It is founded on the fact that, if the border were a line normal to $\nu$, then the function $f_{\nu}^{-1}(\bar{c}(\cdot))$ would be linear on any usable region. Like Algorithm 7.1, it (1) requires that one solve equations $f_{\nu}\left(\sigma_{i}\right)=\bar{c}\left(X_{i}\right)$ for $\sigma_{i}$ (with $f_{\nu}$ the cumulative distribution function for $\nu$-normal lines), (2) will require iteration if $f_{\nu}$ indeed depends on $\nu$ but then is not known to have a solution for all data not from linear borders, and 
(3) reproduces linear borders and yields second-order convergence under appropriate conditions. Unlike Algorithm 7.1, it (4) needs no determination of lines tangent to circles, (5) requires that $\overline{X_{1} X_{2} X_{3}}$ not degenerate - that the ratio of the diameters of its circumscribed to inscribed circles be bounded above-so that Algorithms 7.1 or 11.1 may still be needed for certain orientations of the border in practice, and (6) reduces exactly to the use of linear interpolation of three usefully usable average colors when they are yielded by the specially sensitive circle of Section 6 .

ALGORITHM 12.1. (Using a locally based approximate normal.) Given three usefully usable points $X_{1}, X_{2}$, and $X_{3}$ (Definition 8.2) within $O(h)$ of each other $(h$ the scale in the blurring (4.1)), labeled so that $\bar{c}\left(X_{i}\right)=: \bar{c}_{i}$ is monotone increasing with $i$, and with $\overline{X_{1} X_{2} X_{3}}$ not degenerate,

(1) Determine a unit normal $\nu$ as follows. If two $\bar{c}_{i}$ are equal, $\nu$ is normal to the corresponding line segment and points towards the side of increasing $\bar{c}$ as indicated by the third value. Otherwise, define $\bar{c}:=\left(\bar{c}_{1}+\bar{c}_{3}\right) / 2$. Suppose first that $\bar{c}_{2} \leq \bar{c}$. If $f_{\nu}(3.2),(4.2),(5.1)$, or (6.1) depends on $\nu$, choose $\nu_{0}$ halfway between the two tangents to $\overline{X_{1} X_{3}}$ and $\overline{X_{2} X_{3}}$ directed towards $X_{3}$. Compute $\sigma_{i}:=f_{\nu_{0}}^{-1}\left(\bar{c}_{i}\right), i=1,2,3$, and $\bar{\sigma}:=f_{\nu_{0}}^{-1}(\bar{c})$. For $j=1,2$, set

$$
w_{j}:=\left(\sigma_{3}-\bar{\sigma}\right) /\left(\sigma_{3}-\sigma_{j}\right), \quad \text { and } \quad Y_{j}:=w_{j} X_{j}+\left(1-w_{j}\right) X_{3} .
$$

If $f_{\nu}$ is independent of $\nu$, set $\nu$ to the unit normal $\nu_{1}$ for $\overline{Y_{1} Y_{2}}$ pointing to the side containing $X_{3}$. Otherwise, use $\nu_{0}$ and $\nu_{1}$ to initiate a (here unspecified) iterative procedure intended to produce $\nu_{0}^{(k)} \rightarrow \nu_{1}^{(k)} \rightarrow \nu_{1}^{(\infty)}=: \nu$. If $\bar{c}_{2}>\bar{c}$, work similarly with $\overline{X_{1} X_{2}}$ and $\overline{X_{1} X_{3}}$ (instead of with $\overline{X_{2} X_{3}}$ and $\overline{X_{1} X_{3}}$ ).

(2) With $\sigma_{m}$ a $\sigma_{i}$ of smallest magnitude, set $X:=X_{m}-h \sigma_{m} \nu$.

(3) The line $L_{A}$ through $X$ perpendicular to $\nu$ is the local approximation to the border.

The generalization to $n$ dimensions is evident. Remarks 11.1 (2)-(3) also apply. We now discuss convergence when $\operatorname{diam}\left(\overline{X_{1} X_{2} X_{3}}\right)$ and $h$ are the same order in size:

Proposition 12.1. (Convergence of Algorithm 12.1.) Suppose (1) the border $\beta$ has bounded curvature; (2) $\rho$ (4.1) either has compact support or is an azimuthally symmetric Gaussian; (3) $\overline{X_{1} X_{2} X_{3}}$ is not degenerate and has diameter the same order in size as $h$; and (4) $\bar{c}\left(X_{i}\right)$ is usefully usable (Definition 8.2), $i=1,2,3$.

If $f_{\nu}$ is independent of $\nu$, let $\nu$ be the normal produced by Algorithm 12.1.

If $f_{\nu}$ actually depends on $\nu$ : we shall be content with sketching the application of the implicit function theorem to the present context, guided by its detailed application to Algorithm 7.1 in Proposition 8.1. The equation

$$
F\left(\alpha, \bar{c}_{1}, \bar{c}_{2}, \bar{c}_{3}\right)=G\left(\alpha, f_{\nu(\alpha)}^{-1}\left(\bar{c}_{1}\right), f_{\nu(\alpha)}^{-1}\left(\bar{c}_{2}\right), f_{\nu(\alpha)}^{-1}\left(\bar{c}_{3}\right)\right)=0
$$

determining the angle $\alpha$ that the normal to $\overline{Y_{1} Y_{2}}$ makes with, say, a coordinate axis, is satisfied, for any linear border $L$ which is usable at the three $X_{i}$, by the angle $L$ makes and its average colors $\bar{c}_{L}\left(X_{i}\right)$. So, suppose (5) there is a local tangent line $L_{\nu_{\beta}}$ to $\beta$ such that hypotheses sufficient for the implicit function theorem are satisfied at the usable data for $L_{\nu_{\beta}}$; specifically, suppose $F=0, \partial F / \partial \bar{c}_{i}=O(1), i=1,2,3$, 
and $\partial F / \partial \alpha \neq o(1)$ there-this last now involves four specific first moments (8.7)(8.8) of $\rho$. As for $\beta$ 's data, suppose (6) that the iteration has converged to yield the normal $\nu=\nu(\alpha)$ associated with the unique solution to $F(\alpha, \cdot)=0$ which exists in view of (5) and Remark 8.1 for $h$ small enough.

Then $X$ lies within $O\left(h^{2}\right)$ of $\beta$, and the line $L_{A}$ lies within $O\left(h^{2}\right)$ of $\beta$ throughout any $O(h)$-size neighborhood of $X$.

Proof. By Remark 8.1, the data, i.e., the three average colors $\bar{c}_{i}$ provided, are $O(h)$ perturbations of the usefully usable average colors $\tilde{c}_{i}$ associated with a line $L_{\nu_{\beta}}$ whose point of tangency to $\beta$ is within $O(h)$ of $\overline{X_{1} X_{2} X_{3}}$.

If $f_{\nu}$ depends on $\nu,(5)$ and (6) imply $\nu$ within $O(h)$ of $\nu_{\beta} . X$ (and, locally, $L_{A}$ ) lies within $O\left(h^{2}\right)$ of $\beta$ because of this accuracy of $\nu$, the boundedness of $\left(f_{\nu}^{-1}\right)^{\prime}$ near the usefully usable datum $\bar{c}_{m}$, and Remark 8.1. Knowing $\partial F / \partial \alpha$ suggests Newton's method for (12.1).

Suppose $f_{\nu}=f$ independent of $\nu$. Then we can be more explicit. It suffices, as above, to show $\nu$ within $O(h)$ of $\nu_{\beta}$. The Algorithm now associates $L_{\nu_{\beta}}$ uniquely with the perturbed data $\tilde{c}_{i}$. Because of the positivity and boundedness of $\left(f_{(\cdot)}^{-1}\right)^{\prime}$ on usefully usable data, the $\sigma_{i}$ and $\bar{\sigma}$ exist uniquely and are $O(h)$ perturbations of the corresponding (unique) quantities $\tilde{\sigma}_{i}$ and $\tilde{\sigma}$ associated with $L_{\nu_{\beta}}$; and each $Y_{i}$ and $\tilde{Y}_{i}$ is a convex combination.

As $\overline{X_{1} X_{2} X_{3}}$ has diameter of order $h$ in size and is not degenerate by our definition, its interior angles are bounded away from 0 and $\pi$ and its edges are exactly of order $h$ in length. Because the $X_{i}$ are ordered as in the Algorithm and because the $\tilde{c}_{i}$ are usefully usable, we see from (7.2) that $\tilde{c}_{3} \neq \tilde{c}_{1}+o(1)$ and that $\cos \angle\left(\overline{X_{1} X_{3}}, \nu_{\beta}\right)$ is bounded away from zero. We assume without loss that $\tilde{c}_{2} \leq\left(\tilde{c}_{1}+\tilde{c}_{3}\right) / 2=: \tilde{c}$. Then, in the same fashion, $\cos \angle\left(\overline{X_{2} X_{3}}, \nu_{\beta}\right)$ is also bounded away from zero.

If $\tilde{c}_{2}$ and $\bar{c}_{2}$ lie on the same side of the respective averages $\tilde{c}$ and $\bar{c}$, it follows that the denominators of both weights $\tilde{w}_{j}$ and, hence, both $w_{j}$ are bounded away from zero, so each $w_{j}=\tilde{w}_{j}+O(h)$. If they do not, then $\tilde{c}_{2}$ and $\bar{c}_{2}$ and the two averages are all within $O(h)$ of each other, so are all separated by order 1 from the average colors of $X_{1}$ and $X_{3}$. It follows, in either case, that each $Y_{i}=\tilde{Y}_{i}+O\left(h^{2}\right)$.

It remains to show that $\nu$ is an $O(h)$ perturbation of $\nu_{\beta}$. For this, it now suffices that $\left\|\tilde{Y}_{2}-\tilde{Y}_{1}\right\| \neq o(h)$. But, as the average $\tilde{c}$ is not within $o(1)$ of either $\tilde{c}_{1}$ or $\tilde{c}_{3}$, neither $\tilde{Y}_{1}$ nor $\tilde{Y}_{2}$ are within $o(h)$ of either $X_{1}$ or $X_{3}$. Thus, since $\overline{X_{1} X_{2} X_{3}}$ is not degenerate, $\left\|\tilde{Y}_{1}-\tilde{Y}_{2}\right\| \neq o(h)$.

It is worth mentioning how the plausibility for the case when $f_{\nu}$ depends on $\nu$ is improved by the success of argument when it does not This is based on the fact that, while $F\left(\alpha, x_{1}, x_{2}, x_{3}\right)$ (12.1) clearly changes its functional form from one case to the other, $G\left(\alpha, y_{1}, y_{2}, y_{3}\right)$ does not. The argument for $\nu$-independent $f_{\nu}$ indicates success when $y_{i}=y\left(x_{i}\right)$ for a variety of functions $y$; so it lends credibility to success when $y_{i}=y\left(x_{i}, \alpha\right)$ with $\partial y / \partial x$ not particularly different from successful $d y / d x$. The principal change in this more complex case is the additional contributions of the function $\partial y / \partial \alpha$ to the requirement $\partial F / \partial \alpha \neq 0$; and this indicates it may be necessary to avoid lower-dimensional sets in $\left(\alpha, x_{1}, x_{2}, x_{3}\right)$-space in this more complex case--just as in Proposition 8.1. 
13. Hydrodynamics Briefly Revisited. If the transport equation for the color had constant coefficients, then the spatial average (4.1), of a solution $c$ to the pure initial value problem, would also solve that problem (as the convolution then commutes with the differential operator). This is no longer true if the equation has variable coefficients which, in this context, involve the local fluid velocity, since the convolution of a solution then satisfies a differential equation with additional terms of lower order.

Moreover, the transport of the average color $\bar{c}$ (4.1) is presumably done numerically, with a spatial grid of size, say, $\delta$. If $\delta$ and the scale $h$ in (4.1) are of comparable size, frequency components (of the color $c$ of two regions) that change sign at nearly every mesh point may not be reduced by (4.1) enough for their mishandling by the difference scheme to be irrelevant to subsequent $O\left(\delta^{2}\right)$ accurate border reconstruction. For example, although the interval-averaging of Section 1 annihilates the highest frequency representable on the mesh, it does not significantly reduce the frequency which changes sign at every fourth meshpoint; so the amount of that frequency in $\bar{c}$ there remains of order $\delta$, i.e., of order $h$. Such high frequencies are particularly difficult to treat accurately en masse with locally based numerical transport equations whose action is independent of the discontinuity - in particular, such frequencies then move at speeds whose error is at best bounded. So, errors exceeding the order $h$ errors required for $O\left(h^{2}\right)=O\left(\delta^{2}\right)$ accurate border reconstruction could conceivably accumulate quickly. To maintain better-thanfirst-order (in $\delta$ ) reconstruction longer, one might let $\delta$ be $o(h)$. On the other hand, unless $h=o\left(\delta^{1 / 2}\right)$, the accuracy of the reconstructed border would be first-order in $\delta$ at best (Proposition 9.1). It may take a good deal more thought-and, globallyor discontinuity-based numerical transport - to effectively resolve such practical matters.

In some computational hydrodynamics contexts, the spatial domain is tessellated with noncongruent cells whose average colors are then used for border reconstruction. We make a brief comment concerning the probability of the $o(h)$ accuracy of such reconstruction at the end of Appendix 1.

Finally, two observations are in order: (1) Although our work can contribute to hydrodynamic schemes having improved truncation error, it does so with a stencil which can be different from present algorithms, and this could lead to different stability problems. Moreover, (2) although higher-order truncation can suffice for higher-order convergence (assuming stability and a linear problem), it is not necessary in some contexts; see, e.g., Kreiss, Manteuffel, Swartz, Wendroff, and White [20] for the context of ordinary differential equations (with some reference to partial differential equations), or the finite element literature concerning "lumped mass" schemes.

Appendix 1. $n$ Dimensions. It is worth extending three of the concepts previously considered to $n$ dimensions. The first extension concerns the contents of Section 4.

Assume one is given a probability density $\rho$ now defined on $n$-dimensional Euclidean space $E^{n}$. Define the "average color" of a color function $c$ to be the scaled 
convolution

$$
\bar{c}(x):=\int_{E^{n}} \rho((\xi-x) / h) c(\xi) d^{n} \xi / h^{n} ;
$$

it bears the scale $h$ as a parameter. Associated with $\rho$ is its cumulative distribution function for $\nu$-normal hyperplanes

$$
f_{\nu}(\sigma):=\int_{-\sigma}^{\infty} \int_{H(\nu)} \rho\left(s \nu+x_{n-1}\right) d^{n-1} x d s,
$$

in which $x_{n-1}$ is restricted to $H(\nu)$, the $(n-1)$-dimensional hyperplane through the origin with unit normal $\nu$. When the border is a linear border $H=H_{\nu}, \nu$ points into $\Omega_{1}$-i.e., towards that side of $H$ where $c=1$ - so that still, as above (3.1), $\bar{c}_{H}(x)=f_{\nu}(\sigma)$, with $h \sigma$, the signed distance from $H$ to $x$ as measured along $\nu$, being negative for $x$ in $\Omega_{0}$ and nonnegative, otherwise. Concerning the present analog of (3.1) itself: for $H=H_{\nu}$ to coincide with $\bar{c}_{H}^{-1}\left(f_{\nu}(0)\right)$ (and not be just a proper subset), one must assume that $f_{\nu}$ is strictly monotone at $\sigma=0$. We shall, in fact, impose a stronger extension of this: Let $M_{0}(\sigma, \nu):=\left(\partial f_{\nu} / \partial \sigma\right)(\sigma, \nu)$ be the mass of the hyperplane (induced by the restriction to it of $\rho$ ) which is normal to $\nu$ and passes through the point $\left(-\sigma \nu_{1}, \ldots,-\sigma \nu_{n}\right)$. Then we assume, for each $\nu$, that the interior of the support of $M_{0}(\cdot, \nu)$ is a nontrivial interval which contains 0 and on which $M_{0}(\cdot, \nu)$ is positive. In particular, then, $f_{\nu}(0)>0$; and $f_{\nu}(0)=1 / 2$ if $\rho$ is symmetric under reflection through the origin.

This last points out that the origin can be placed at a special point in some (but not all) mass distributions so that every hyperplane through the origin divides the distribution into two pieces of equal mass. The analog of (3.1) is complete for such distributions when the origin is so placed-in that, then, $H=\bar{c}_{H}^{-1}(1 / 2)$. Further discussion of such halfway points for mass distributions will be found in Beyer and Swartz [1].

As a practical example of a cumulative distribution function (A1.2) for $\nu$-normal hyperplanes, we outline in Appendix 4 an algorithm to compute the $n$th degree polynomial spline function which is the distribution function associated with a union (presumed to have unit mass) of $n$-dimensional, individually homogeneous tetrahedra (simplices) having nonoverlapping interiors.

Next we derive the integral equation for the azimuthally symmetric density $\rho_{n}(r)$, $r:=\sqrt{x_{1}^{2}+\cdots+x_{n}^{2}}$, on the $n$-ball of diameter one which yields (6.1) as the now $\nu$-independent cumulative distribution function (A1.2) for $\nu$-normal hyperplanes of dimension $n-1$. Let $S_{k}(\xi):=2 \pi^{k / 2} \xi^{k-1} / \Gamma(k / 2)$ be the $(k-1)$-measure of the surface of the $k$-ball of radius $\xi$. Then $\rho_{n}$ is now related to the rate of change of $f$ as follows: For $0 \leq \sigma<1 / 2$ (and compare (6.2))

$$
(d f / d \sigma)(\sigma)=1=\int_{0}^{\sqrt{1 / 4-\sigma^{2}}} \rho_{n}\left(\sqrt{\sigma^{2}+z^{2}}\right) S_{n-1}\left(\sqrt{\sigma^{2}+z^{2}}\right) d z .
$$

Hence, from $(6.3), \rho_{n}(r):=2 /\left(\pi S_{n-1}(r) \sqrt{1 / 4-r^{2}}\right)=2 \rho(r) / S_{n-1}(r)$ has the same type of singularity at the outer surface of the ball that $\rho$ had in Section 6 .

Next, we briefly consider the classification of $(n-1)$-dimensional hyperplanes (henceforth, simply "hyperplanes") simultaneously tangent to $n$ balls in $n$-space, 
assuming their centers determine a unique hyperplane-but supply a bit more detail only for the potentially practical case $n=3$. Algorithms determining such hyperplanes are also proposed.

For $n=2$ (Section 7) the above assumption eliminated the case of concentric discs and the accompanying possibility of infinitely many mutually tangent lines when the radii were equal. Moreover, there were then from zero to four mutually tangent lines - and each was the reflection of another in the line $L_{0}$ which contains the segment $\overline{X_{1} X_{2}}$ joining the two centers.

Some of this remains true for $n$-balls with centers $X_{1}, \ldots, X_{n}$ and corresponding radii $r_{1}, \ldots, r_{n}$. Thus, the reflection of a mutually tangent hyperplane in the hyperplane $H_{0}$ determined by the convex hull $\overline{X_{1} \ldots X_{n}}$ of $X_{1}, \ldots, X_{n}$ is also mutually tangent. But, there can be at least $2^{n}$ distinct solutions, as is exemplified for $n=3$ by placing the 1-, 2-, and 3-balls at the corners of the rack-whence the surface of the pool table is one solution; there is one solution under the 1-ball and the 2-ball but over the 3-ball; and, two more like it by symmetry; and the reflection of these four solutions in the plane of the balls' centers gives the total of 8 solutions.

There can be fewer, however-from 7 down to none. As the 3-ball rolls to become tangent to the circular cylinder $C_{12}$ between its circles of tangency with the 1- and 2-balls, a pair of solutions coalesce (7). As the 2-ball rolls through $C_{12}$ to become tangent to the similar cylinder $C_{13}$ containing the 1- and 3-balls, another pair coalesce (6). And the third pair coalesce when each ball is tangent to the cylinder $C_{23}$ containing the other two (5). In each of these situations, if the rolling ball moves inside the cylinder it is approaching, the coalescent pair disappears $(6$ differently, 5 differently, etc.) until one is left with only the 2 parallel to the table. Other situations result when $\overline{X_{1} X_{2} X_{3}}$ is a right triangle. Reducing, now, the radius of the 3-ball, one has the following example of exactly 1 mutually tangent plane: let the smaller 3-ball (a) lie on the same side of both the 1- and 2-balls and (b1) be internally tangent to $C_{12}$. And, there are 0 mutually tangent planes if, instead, (b2) the closure of this smaller 3-ball is contained in the interior of $C_{12}$.

The $2^{n}$ distinct hyperplanes mutually tangent to $n$ balls in $n$-space is exemplified by $\overline{X_{1} \ldots X_{n}}$ being a regular tetrahedron (simplex) in the hyperplane $x_{n}=0$; equal and sufficiently small radii for the balls; with the tangency for the $i$ th ball occurring either for $x_{n}>0$ or $x_{n}<0$, and this last being independent of such locations on the other balls.

That there are at most $2^{n-k}$ distinct hyperplanes tangent to $n$ balls in $n$-space when (a) the centers $X_{1}, \ldots, X_{n}$ determine a unique hyperplane, and (b) exactly $k$ of the corresponding radii $r_{1}, \ldots, r_{n}$ are zero, follows from the character of an algorithm one may use to find the planes. The equation for the points $y$ in a hyperplane in terms of its unit normal $\nu$ and its signed distance $\Delta$ (measured along $\nu$ with its foot resting on the hyperplane) from some origin - which we now take to be the mean of the $X_{i}^{\prime} s: 0=\left(X_{1}+\cdots+X_{n}\right) / n$-is

$$
(y+\Delta \nu)^{T} \nu=0
$$

Consequently, with $X$ the $n \times n$ matrix whose $j$ th column is $X_{j}$, assumption (a) above means that the system to be solved for the pair $\left(\nu_{0}, \Delta_{0}\right)$ associated 
with the hyperplane determined by the balls' centers, namely

$$
X^{T} \nu_{0}=0, \quad \nu_{0}^{T} \nu_{0}=1, \quad\left(\Delta_{0}=0\right)
$$

has exactly one solution (modulo $\nu_{0}$ 's sign). Moreover, since the origin is the average, the span of $1:=(1, \ldots, 1)^{T}$ is the nullspace of $X$ and hence is the set of vectors orthogonal to the range of $X^{T}$. Consider the $Q R$ decomposition of $X^{T}$ (cf. Golub and Van Loan [15]): There is a permutation matrix $P$ such that $X^{T} P=Q R$ where $Q$ has orthonormal columns and $R$ is upper triangular. Since by assumption $X$ has rank $n-1$, the typical $Q R$ computer code will yield $P$, the first $n-1$ columns of $Q$, and $R$ (with its first $n-1$ diagonal elements nonzero). We know, however, that the last column of $Q$ is $Q_{n}=1 / \sqrt{n}$. Then the solution to (A1.4), i.e., to $Q R P^{T} \nu_{0}=0$, is found by

$$
\left(\hat{\nu}_{0}\right)_{n}:=1, \quad R \hat{\nu}_{0}=0, \quad \tilde{\nu}_{0}:=\hat{\nu}_{0} /\left\|\hat{\nu}_{0}\right\|, \quad \nu_{0}:=P \tilde{\nu}_{0}
$$

via a simple backsolve. The unit vector $\nu_{0}$ now spans the nullspace of $X^{T}$.

To continue development of the algorithm: A point $X_{i}$ is at the signed distance $a_{i}$ (measured, like $\Delta$ was, along $\nu$ ) from a hyperplane (A1.3) if and only if $X_{i} \cdot \nu+$ $a_{i}+\Delta=0$ (independent of the origin's location). Hence, with

$$
a:=\left( \pm r_{1}, \ldots, \pm r_{n}\right)^{T}
$$

the given $n$-vector of signed distances, the pairs $(\nu, \Delta)$ associated with the desired mutually tangent hyperplanes coincide with the solution set of the $n+1$ equations

$$
X^{T} \nu=-(a+\Delta \mathbf{1})=Q R P^{T} \nu, \quad \nu^{T} \nu=1 .
$$

No solution need exist; but if one does, then at most two do; and each can be found as follows. Since 1 spans the orthogonal complement of the range of $X^{T}$, set

$$
\Delta:=-a^{T} 1 / \sqrt{n}
$$

so that $a+\Delta 1$ is in the range of $X^{T}$. Let $R_{t}$ be $R$ with its last row and column truncated, so that $R_{t}$ is $(n-1) \times(n-1)$, upper triangular, and nonsingular. Multiplying (A1.6) by $Q^{T}$, one sees it appropriate to solve

$$
R_{t} \hat{\nu}=-\left(Q_{1}^{T} a, \ldots, Q_{n-1}^{T} a\right)^{T}
$$

for the $(n-1)$-vector $\hat{\nu}$; and, finally, to find the unit $n$-vector

$$
\nu:=\gamma \nu_{0}+P\left(\hat{\nu}^{T}, 0\right)^{T}
$$

by first solving the necessary quadratic equation $\left(P^{T} \nu\right)^{T}\left(P^{T} \nu\right)-1=0$, i.e.,

$$
\gamma^{2}+2\left(\left(\hat{\nu}^{T}, 0\right) \tilde{\nu}_{0}\right) \gamma+\left(\hat{\nu}^{T} \hat{\nu}\right)-1=0
$$

for $\gamma$. We will have found the (at most two) solutions of (A1.6) to the extent that (A1.10) has real solutions $\gamma$. This completes

Algorithm A 1.1. (For hyperplanes simultaneously tangent to $n$ balls in $n$ space, the $j$ th ball with center $X_{j}$ and radius $r_{j}$, and assuming that $X_{1}+\cdots+X_{n}=$ 0 with $\operatorname{dim}\left(\operatorname{span}\left(X_{1}, \ldots, X_{n}\right)\right)=n-1$.) (T. Manteuffel, Los Alamos National Laboratory).

(1) With $X:=\left(X_{1}, \ldots, X_{n}\right)$, decompose $X^{T} P=Q R$, yielding the permutation matrix $P$, orthonormal columns $Q_{1}, \ldots, Q_{n-1}$, and upper triangular $R$ having rank $n-1$ and last row zero. 
(2) Solve (A1.4) via (A1.5), yielding unit vectors $\tilde{\nu}_{0}$ and $\nu_{0}$.

(3) With $1:=(1, \ldots, 1)^{T}$ and for each vector $a:=\left( \pm r_{1}, \ldots, \pm r_{n}\right)^{T}$ of signed distances, solve (A1.6) via (A1.7)-(A1.10), yielding at most two pairs $(\nu, \Delta)$, and thus at most two associated hyperplanes (A1.3) tangent to the $n$ balls at signed distance $a_{j}$ from $X_{j}, j=1, \ldots, n$, as measured along $\nu$.

There are $2^{n-k}$ distinct possibilities here for $a$ if exactly $k$ of the radii $r_{i}$ are zero, and the quadratic equation (A1.10) yields another factor of two for the number of possible solutions $(\nu, \Delta)$. But, each hyperplane has two normals; so the desired conclusion of at most $2^{n-k}$ possible hyperplanes follows.

If it is known that the balls' centers $X_{1}, \ldots, X_{n}$ comprise a linearly independent set of vectors, then an apparently simpler algorithm to solve (A1.6)-its first and final equalities being independent of the origin's location-can be set down. (This is the case in the typical application contemplated in this paper, when the $X_{j}$ would be $n$ of the $2^{n}$ vertices of an $n$-cube, so that one could pick, as the origin $X_{0}=0$, one of the other vertices of that $n$-cube. This choice for $X_{0}$ is not arbitrary unless $n=2$, since $n+1$ vertices can lie in a hyperplane for $n>2$. However, it is a relatively easy choice to make if $n=3$. Alternatively for $n=3$, one could move the mean by the vector product-nonzero by assumption (a) but suitably normalized - of a pair of differences.)

AlgORITHM A 1.2. (For hyperplanes simultaneously tangent to $n$ balls in $n$-space as before, except that $X_{1}, \ldots, X_{n}$ are known to be linearly independent vectors.)

(1) With $X, a$, and 1 as in Algorithm A1.1, find $b$ and $q$ such that $X^{T} b=-a$ and $X^{T} q=-1$.

(2) Solve the quadratic equation

$$
\left(q^{T} q\right) \Delta^{2}+2\left(q^{T} b\right) \Delta+\left(b^{T} b\right)-1=0 \quad \text { for } \Delta .
$$

(3) For each real $\Delta$, set $\nu=b+\Delta q$.

The circumstances under which there exist no solutions seem clear, at least for $n=3$. First, there exists no solution if one closed ball is in the interior of another. Otherwise, for any two balls with centers $X_{1}$ and $X_{2}$, let $T_{\text {ext }}$ be the (doubly) infinite cone (or cylinder) of mutually tangent lines whose vertex does not lie on the segment $\overline{X_{1} X_{2}} . T_{\text {ext }}$ divides 3-space into three open, connected components, of which two are convex cones - unless the radii are the same when, as on the pool table, the two open convex cones become the interior of a cylinder. Similarly, let $T_{\text {int }}$ be the doubly infinite cone (it may be a plane or may not exist) of mutually tangent lines whose vertex lies on the segment $\overline{X_{1} X_{2}}$; it defines two more open convex cones when it exists. The original two open cones (or cylinder), or their intersection with the second pair of cones when available, yields one (usually two) open convex body(s). There will exist no mutually tangent planes to a third ball if and only if its closure is contained in either of these body(s).

As is true for the analog in two dimensions, the distance of a plane from four noncoplanar points usually determines it; but, given also four arbitrary distances, there will generally not exist a corresponding plane. 
Finally, should the probability density $\rho$ or the scale $h$ used in the blurring vary from data point $X_{i}$ to data point $X_{j}$ (for example, in the context described in Section 13), our algorithms can continue to make sense. For Algorithm 7.1 in its $n$-dimensional form: For each $i$ let $\rho_{i}(\cdot)$ be a probability distribution and $h_{i}>0$ be a scale. Then, for each fixed normal $\nu$, the level surfaces for the average coloring $\bar{c}_{i, H_{\nu, 0}}$ of a half space $\mathscr{H}_{1}$, bordered by the hyperplane $H_{\nu, 0}$ through the origin, are still hyperplanes parallel to $H_{\nu, 0}$, since (from (A1.1))

$$
\bar{c}_{i, H_{\nu, 0}}(x)=\int_{\mathscr{H}_{1}} \rho_{i}\left((\xi-x) / h_{i}\right) d^{n} \xi / h_{i}^{n}=f_{i, \nu}(\sigma),
$$

with $\sigma=x \cdot \nu / h_{i}$ the signed distance from $H_{0, \nu}$ to $x$ scaled by $h_{i}$, and with $f_{i, \nu}$ the cumulative distribution function (A1.2), under $\rho_{i}$, for $\nu$-normal hyperplanes. So, if $\bar{c}_{i}:=\bar{c}_{H_{\nu}}\left(X_{i}\right), i=1, \ldots, n$, are $n$ usable values of the average color, the $i$ th under $\rho_{i}$ with scale $h_{i}$, of a half-space bordered by $H_{\nu}$, then the distance of $X_{i}$ from $H_{\nu}$ is $r_{i}(\nu):=h_{i}\left|f_{i, \nu}^{-1}\left(\bar{c}_{i}\right)\right|$ (compare (7.3a)). Hence, $H_{\nu}$ is again simultaneously tangent to $n$ balls, now of radii $r_{1}(\nu), \ldots, r_{n}(\nu)$, centered, respectively, on $X_{1}, \ldots, X_{n}$. It follows that Algorithms A1.1 and A1.2 could be useful in an iteration to determine $\nu$ and $H_{\nu}$ in the $n$-dimensional analog of Algorithm 7.1.

We expect that the proofs of local existence, stability, and $o\left(\max \left(h_{1}, \ldots, h_{n}\right)\right)$ convergence in Sections 8 and 9 (for $n=2$ ) will generalize for such densities $\rho_{i}$ and scales $h_{i}$. For example, consider the stability result, Proposition 8.1. The analog of (8.6) is now

$$
F\left(\alpha, \bar{c}_{1}, \bar{c}_{2}\right):=\cos \alpha-\left(h_{2} f_{2, \nu(\alpha)}^{-1}\left(\bar{c}_{2}\right)-h_{1} f_{1, \nu(\alpha)}^{-1}\left(\bar{c}_{1}\right)\right) / \delta=0,
$$

so that, using (8.7), the condition via the implicit function theorem for the existence, uniqueness, and uniform stability of $\tilde{\alpha}=\alpha\left(\tilde{c}_{1}, \tilde{c}_{2}\right)$ solving this near a solution $\left(\alpha_{0}, \bar{c}_{1}, \bar{c}_{2}\right)$ is that

$$
\partial F / \partial \alpha=\left(h_{2} \mu_{2}^{(1)}-h_{1} \mu_{1}^{(1)}\right) / \delta-\sin \alpha \neq o(1),
$$

in which $\mu_{i}^{(1)}:=\mu_{i}^{(1)}\left(\sigma_{i}, \nu\right)$, with $\mu_{i}^{(1)}(\sigma, \nu)$ again being $\rho_{i}$ 's specific first moment for $\nu$-normal lines about $P_{\sigma, \nu}(8.7)-(8.8)$, and with $\sigma_{i}:=f_{i, \nu}^{-1}\left(\bar{c}_{i}\right)$. The analog of (8.4) is (from (A1.12) and assuming $h_{2} \sigma_{2} \neq h_{1} \sigma_{1}$ )

$$
\cos \alpha\left(h_{2} \mu_{2}^{(1)}-h_{1} \mu_{1}^{(1)}\right) /\left(h_{2} \sigma_{2}-h_{1} \sigma_{1}\right)-\sin \alpha \neq o(1) .
$$

The analog of Algorithm 11.1-involving now three points $Z_{1}, Z_{2}$, and $Z_{3}:=Y$ with their three associated cumulative distributions $f_{i, \nu}$ and scales $h_{i}$-should not only work (using $h_{i} f_{i, \nu}^{-1}$ for $h f_{\nu}^{-1}$ there as appropriate) but also converge as in Section 11 if the three scales are of comparable small size.

Algorithm 12.1 extends as follows: The three average colors $\bar{c}_{i}$ (with associated cumulative distributions $f_{i, \nu}$ and scales $h_{i}$ ) may no longer order the $X_{i}$ appropriately - but (having picked an initial normal $\nu_{0}$ ) the values $\bar{r}_{i}:=h_{i} \sigma_{i}$, with $\sigma_{i}:=f_{i, \nu_{0}}^{-1}\left(\bar{c}_{i}\right)$, still do. We shall suppose that not all three $\bar{r}_{i}$ have the same sign and that the magnitude of the singleton is not much smaller than that of its largest opposite number. Then, for the singleton and one of its two opposite numbers: compute the weight for linearly interpolating 0 between the two associated $\bar{r}_{i}$, and use it to interpolate linearly between the two corresponding $X_{i}$, thereby obtaining 
$Y_{1}$. Next, use the other to similarly obtain $Y_{2}$-and let $\nu_{1}$ be the unit normal for the segment $\overline{Y_{1} Y_{2}}$. Iterate as necessary to converge on $\nu$. The line now containing $\overline{Y_{1} Y_{2}}$ is the approximate border $L_{A}$ associated with the extension of Algorithm 12.1.

The original version of this work (Swartz [24]) anticipated $o(h)$ accuracy only for densities $\rho_{x}(\xi)$ which varied smoothly from $x=X_{i}$ to $x=X_{j}$ (uniformly in $h$ and $\delta$ ); but it is now (May, 1988) clear, in view of (A1.12)-(A1.14), that smoothness is not necessary.

Appendix 2. Locating Linear Borders Relative to Two Usable Points: General $f_{\nu}$. When the cumulative distribution function $f_{\nu}$ for $\nu$-normal lines (4.2) actually depends on $\nu$, an important practical problem is the existence of solutions to (7.2) when the usable data $\left(X_{1}, \bar{c}_{1}\right)$ and $\left(X_{2}, \bar{c}_{2}\right), X_{1} \neq X_{2}$, is not known in advance to be associated with some line. ${ }^{\dagger \dagger}$ Proof will be provided momentarily that either of the following conditions suffice for existence:

$$
\sup _{\nu} \mid\left(f_{\nu}^{-1}\left(\bar{c}_{2}\right)-f_{\nu}^{-1}\left(\bar{c}_{1}\right) \mid \leq \delta / h\right.
$$

or

$$
\left|\bar{c}_{2}-\bar{c}_{1}\right| / \min _{\nu} \min _{\sigma \text { usable }}\left(D f_{\nu}\right)(\sigma) \leq \delta / h
$$

where $D f_{\nu}(\sigma)$ is the amount of the density $\rho$ on the line $L_{\nu}$ at a (signed) distance $\sigma$ from the origin. (If $\min \left(D f_{\nu}\right)$ is too small, then the meaning of "usable" can be changed throughout - to be that associated with values of $\bar{c}$ being in $[\varepsilon, 1-\varepsilon]$, some suitable $0<\varepsilon<1 / 2$.)

Other sufficient conditions can be given, especially if more is known about $\rho$ and the data. For example, if $\rho$ is symmetric under reflection through the origin, then, for each $\nu$, the graph of $f_{\nu}$ is symmetric under reflection through $(0,1 / 2)$. If, also for each $\nu$, the amount of $\rho$ on $L_{\nu}$ does not increase as the distance $|\sigma|$ of $L_{\nu}$ from the origin increases (as it would if $\rho$ were constant on its support, and that support were both symmetric through the origin and convex), then a sufficient condition for existence for usable data embracing $1 / 2$, i.e., satisfying $\bar{c}_{1} \leq 1 / 2 \leq \bar{c}_{2}$ or $\bar{c}_{2} \leq 1 / 2 \leq \bar{c}_{1}$, would be

$$
d\left|\bar{c}_{2}-\bar{c}_{1}\right| \leq \delta / h
$$

here $d$ is the supremum (over $\nu$ ) of the diameter of the set of usable arguments $\sigma$ for $f_{\nu}$. (The slope of a secant line for any such $f_{\nu}$, with $f_{\nu}\left(\sigma_{1}\right) \leq 1 / 2 \leq f_{\nu}\left(\sigma_{2}\right)$ and $\sigma_{1}<\sigma_{2}$, is not less than $1 / d$; and the case $\bar{c}_{1}=1 / 2=\bar{c}_{2}$ is trivial.)

In particular, (A2.3) is sufficient for the blurring associated with the average color of a mesh square in Sections 13 . Hence, if three usable vertices of a mesh

${ }^{\dagger \dagger}$ When the average colors are both $1 / 2$, existence is given by the "ham-sandwich theorem" of Steinhaus, which concerns the simultaneous bisection of two measurable sets by a line. For prescribed portions other than $1 / 2$, portions which might be different from each other (and were their locations with respect to the dividing line immaterial), existence would seem to be provided by De Cecco [12] except that his result is not true in the generality he states (Fenske's [14] review, saying that De Cecco [12] is a correct but trivial extension, is unfortunate). One set of counterexamples is associated with the nonexistence part of Summary 7.1 e.g., let the sets be translates of each other by sufficiently short distances and the reader will find others. My favorite counterexample spotted by the Los Alamos physicist L. Heller during lunch concerns "soup". 
square have data which is compatible with the data associated with some line-at least, to the extent that, with $X_{2}$ at the right-angle, (a) $\bar{c}_{2}$ does not lie between the other two $\bar{c}_{i}$, and (b) at least one, call it $\bar{c}_{1}$, of the other two lies on the side opposite $1 / 2$ from $\bar{c}_{2}$-then (A2.3), with $d=\sqrt{2}$, can be used to ensure existence of a solution to (7.2) (or for the other data pair whose average colors embrace 1/2).

(A2.1), (A2.2), or (A2.3) are sufficient conditions (in their respective contexts) for the existence of solutions to (7.2) by the following continuity argument. Recall the circles $C_{i}(\nu)(7.3 \mathrm{~b})$ centered on $X_{i}$ with radii $r_{i}(\nu):=h\left|f_{\nu}^{-1}\left(\bar{c}_{i}\right)\right|(7.3 \mathrm{a})$.

Let $\alpha:=L_{\tau, \nu}$. Suppose first that $\bar{c}_{1} \leq \bar{c}_{2}$. Each of (A2.1), (A2.2) or (A2.3) ensures that, for any $\nu=\nu(\alpha)$, the right-hand side of $(7.2)$ lies in $[0,1]$. Hence, its arccosine defines two functions $\gamma_{i}(\alpha)$, on $[(i-1) \pi / 2, i \pi / 2]$, to itself $-i=1$ or 4 being the quadrant in which $(1, \alpha)$ lies in suitable polar coordinates. We argue in detail only about $\gamma_{1}$. Since each $f_{\nu(\alpha)}^{-1}\left(\bar{c}_{i}\right)$ is a continuous function of $\alpha, \gamma_{1}$ is, too; and we need show only that if $\gamma_{1}(\alpha)=\alpha$, then we have a solution. So, with $\gamma_{1}(\alpha)=\alpha$ fixed in $[0, \pi / 2]$, set $\nu:=\nu(\alpha)$. There are three cases. (1) Suppose $\bar{c}_{2} \leq f_{\nu}(0)$. Then $r_{1}(\nu) \geq r_{2}(\nu)$, and there is a solution-specifically, a line $L_{\nu}$ mutually tangent to the two circles, with $\nu$ (on $L_{\nu}$ ) pointing away from both $X_{i}$. (2) Suppose $\bar{c}_{1} \leq f_{\nu}(0)<\bar{c}_{2}$. Then there is a solution-specifically, a mutually tangent line with $\nu$ pointing away from $X_{1}$ and towards $X_{2}$. (3) Suppose $f_{\nu}(0) \leq \bar{c}_{1}$. Then $r_{1}(\nu) \leq r_{2}(\nu)$, and there is a solution-specifically, a mutually tangent line with $\nu$ pointing towards both $X_{i}$.

We conclude that if $\bar{c}_{1} \leq \bar{c}_{2}$, then there is a solution with $\alpha$ in $[0, \pi / 2]$, and (arguing with $\gamma_{4}$ ) another in $[3 \pi / 2,2 \pi]$. The two are distinct unless $\gamma_{1}(0)=0$ and $\gamma_{4}(2 \pi)=2 \pi$.

Finally, for $\bar{c}_{1} \geq \bar{c}_{2}$ : the right-hand side of (7.2) now lies in $[-1,0]$. The argument is similar, but one works with $\gamma_{2}$ and $\gamma_{3}$.

SUmmary A 2.1. (Compare Summary 7.1.) For $f_{\nu}$ dependent on $\nu$ : Conditions (A2.1), (A2.2), or (A2.3) suffice (in their respective contexts) for the existence of at least one oriented line $L_{\nu}$ solving (7.2). The component $\nu_{\|}$of $\nu$ along $\overline{X_{1} X_{2}}$ $i s$ in the direction of increasing $\bar{c}$ for any solution. There will be at least two solutions-- not necessarily reflections but still one with $\nu_{\perp}$ on one side of $\overline{X_{1} X_{2}}$ and a second, on the other side unless $(a) \bar{c}_{1}$ and $\bar{c}_{2}$ lie on the same side of $f_{ \pm \tau}(0)$ and $\left|r_{2}( \pm \tau)-r_{1}( \pm \tau)\right|=\delta$, or $(b) \bar{c}_{1}$ and $\bar{c}_{2}$ embrace $f_{ \pm \tau}(0)$ and $r_{1}( \pm \tau)+r_{2}( \pm \tau)=\delta$.

As for necessary conditions: in view of (7.2) and the mean value theorem, replace "sup" with "inf" and/or "min" with "max" in (A2.1) or (A2.2). In particular, there always exist usable numbers $\bar{c}_{1}$ and $\bar{c}_{2}$ which are too far apart, relative to small $\left\|X_{2}-X_{1}\right\| / h$, for any linear border to yield such an increase in such a short distance. For the specially sensitive disc yielding the $\nu$-independent, linear (where usable) cumulative distribution function $f_{\nu}=f(6.1)$, all five conditions coincide with $\left|\bar{c}_{2}-\bar{c}_{1}\right| \leq \delta / h$.

Existence and uniqueness for data near linear-border data is considered in Proposition 8.1. We do not develop specific algorithms for solutions of (7.2) when $f_{\nu}$ is truly $\nu$-dependent, but note that Algorithm A1.2 could be useful in iteration. 
Appendix 3. The Angle Between a Circle and a Small Nearby Line Segment. It suffices to prove (9.4) in the context of $\beta$ 's osculating disc halfway between $X_{\beta, j}$ and $X_{\beta, j+1}$.

Thus, suppose $h \rightarrow 0$, that $C$ is a circle of curvature $\kappa$ (there, $\kappa_{\beta, j+1 / 2}$ ), and that distinct points $X_{1}=X_{1}(h)$ and $X_{2}=X_{2}(h)$ (there, $X_{j}$ and $X_{j+1}$ ) converge to a point $c$ on $C$. Let $\delta:=\left\|X_{2}-X_{1}\right\|=O(h)$, and $\tau:=\left(X_{2}-X_{1}\right) / \delta$. Let $Y_{1}$ and $Y_{2}$ (there, $X_{\beta, j}$ and $X_{\beta, j+1}$ ) be the closest points on $C$ to $X_{1}$ and $X_{2}$, respectively. With $\nu_{i}$ the unit normal to $C$ at $Y_{i}$ pointing toward $C$ 's center, set $\sigma_{i}:=\nu_{i} \cdot\left(X_{i}-Y_{i}\right) / h$, and presume it bounded. Let $\nu$ be the unit normal to $\overline{Y_{1} Y_{2}}$ (also pointing toward $C$ 's center). We wish to prove the present analog of (9.4), namely, that

$$
\left(\sigma_{2}-\sigma_{1}\right) h / \delta=\tau \cdot \nu\left(1+O\left(\kappa^{2} \delta^{2}\right)\right)
$$

Now, $\left(X_{i}-Y_{i}\right)=h \sigma_{i} \nu_{i}$, so

$$
\left(\nu-\nu_{i}\right) \cdot\left(X_{i}-Y_{i}\right)=\left(1-\nu \cdot \nu_{i}\right) h \sigma_{i}=h \sigma_{i} \cdot O\left(\delta^{2} \kappa^{2}\right)
$$

since $\nu \cdot \nu_{i}=\cos \alpha$ with $\sin \alpha=O\left(\kappa\left\|Y_{2}-Y_{1}\right\|\right)=O\left(\kappa\left\|X_{2}-X_{1}\right\|[1+O(h)]\right)=$ $O(\kappa \delta)$. With this,

$$
\begin{aligned}
\left(\sigma_{2}-\sigma_{1}\right) h & =\nu_{2} \cdot\left(X_{2}-Y_{2}\right)-\nu_{1} \cdot\left(X_{1}-Y_{1}\right) \\
& =\nu \cdot\left(X_{2}-X_{1}\right)-\left(\nu-\nu_{2}\right) \cdot\left(X_{2}-Y_{2}\right)+\left(\nu-\nu_{1}\right) \cdot\left(X_{1}-Y_{1}\right) \\
& =\nu \cdot \tau \delta-\left(1-\nu \cdot \nu_{2}\right) h \sigma_{2}+\left(1-\nu \cdot \nu_{1}\right) h \sigma_{1} \\
& =\nu \cdot \tau \delta-\left(\sigma_{2}-\sigma_{1}\right) h \cdot O\left(\kappa^{2} \delta^{2}\right),
\end{aligned}
$$

and (A3.1) follows.

Appendix 4. The Cumulative Distribution Function of Polyhedra. (November, 1987). We review first a definition, equivalent to Curry and Schoenberg's [10], of a polynomial B-spline - the one normalized so that its integral over its support is one. For $n \geq 2$, let $S$ be a nondegenerate $n$-dimensional simplex with vertices $\left(v_{i}\right)_{0}^{n}$, and let $l_{\nu}$ be a line through the origin in the direction of the unit $n$-vector $\nu$. Let $t_{i}=\nu \cdot v_{i}$ be the signed distance from the origin of the orthogonal projection $P_{\nu} v_{i}$ of $v_{i}$ onto $l_{\nu}$. Now, reorder the $t_{i}$ so that the $(n+1)$-sequence $T(S, \nu):=\left(t_{0}, \ldots, t_{n}\right)$ is monotone nondecreasing. Then the value, at the real number $\xi$, of the ( $n$ th-order, univariate, polynomial) B-spline $M_{T(S, \nu)}$ with knots $T(S, \nu)$ is the ratio of the $(n-1)$-dimensional volume of a linear section of $S$ - the one contained in the $(n-1)$-dimensional hyperplane $H_{\nu}(\xi):=\{x: \nu \cdot x=\xi\}$ normal to $\nu$ and intersecting $l_{\nu}$ at the signed distance $\xi$ from the origin-to the ( $n$-dimensional) volume of $S$ itself. (This geometric notion has been extended to create multivariate simplicial B-splines (de Boor [4]) and other types of multivariate B-splines-see, e.g., Höllig [16].)

Consequently, the cumulative distribution function for $\nu$-normal hyperplanes (A1.2), associated with the (constant) density $\rho$ of a homogeneous simplex $S$ of mass one, is

$$
f_{\nu}(\sigma ; S):=\int_{-\sigma}^{\infty} M_{T(S, \nu)}(\xi) d \xi
$$


Moreover, the cumulative distribution function associated with the union $U$ of individually homogeneous simplices $S_{j}$ - of mass $w_{j}\left(\sum_{j} w_{j}=1\right)$, and who's interiors do not overlap-is $f_{\nu}(\sigma ; U):=\sum_{j} w_{j} f_{\nu}\left(\sigma ; S_{j}\right)$. Such functions are therefore univariate, polynomial splines of order $n+1($ degree $n)$ whose $\operatorname{knot}$ set $T(U, \nu)$ is a subset of the (reordered) projections of all of the vertices involved. Note, however, that (a) $T(U, \nu)$ may be a proper subset of all the vertex projections; and that (b) although one can associate a sequence of $n$ th-order B-splines with the natural ordering of $T(U, \nu)$ and then associate a simplex with each of these B-splines, the union of the closed simplices so defined need not coincide with $U$.

As for a more specific algorithm: Because some of the $n+1$ knots in $T(S, \nu)$ may coincide or be close to coinciding (although no knot has multiplicity exceeding $n$ as $S$ is nondegenerate), it seems wise to utilize the widely available computer programs (e.g., in the IMSL or SLATEC libraries) that de Boor [2], [5] created, based on algorithms (de Boor [3], see also Cox [9]) developed for the stable (Cox [9]) computation of B-splines. For this, recall that with any nondecreasing biinfinite knot sequence $\left(t_{i}\right)_{i=-\infty}^{\infty}$ (in which at most $k$ elements coincide) there is associated the bi-infinite sequence of $k$ th-order B-splines $\left(B_{i, k}\right)_{-\infty}^{\infty}$ (each with its knots $t_{i} \leq \cdots<\cdots \leq t_{i+k}$, and normalized so that $\left.\sum_{i} B_{i, k}(x) \equiv 1\right)$. $B_{i, k}$ is positive on $\left(t_{i}, t_{i+k}\right)$ and vanishes outside $\left[t_{i}, t_{i+k}\right]$; so that, if $t_{L}<t_{L+1}$ and $t_{R-1}<t_{R}$, the only B-spline coefficients relevant to the value of a $k$ th-order spline $s=\sum_{i} c_{i} B_{i, k}$ on $\left(t_{L}, t_{R}\right)$ are $c_{L-k+1}, \ldots, c_{R-1}$.

From de Boor's book [5, p.109], $M_{T(S, \nu)}=n B_{0, n} /\left(t_{n}-t_{0}\right)$. Thus, integrating the divided difference definition of $B_{0, n}$ (ibid., p.108), we find $f_{\nu}$ (A4.1) to be given by

$$
f_{\nu}(\sigma ; S)=\left[t_{0}, \ldots, t_{n}\right](\cdot+\sigma)_{+}^{n}
$$

in which $(y)_{+}^{n}$ is (the "positive part" of $\left.y\right)^{n}$, and the $n$ th-order divided difference operator $\left[t_{0}, \ldots, t_{n}\right]$ is defined even for coalescent cases (ibid., p.10). (A4.2) is tempting for parallel computation since its evaluation does not require $t_{0}, \ldots, t_{n}$ to be ordered and since $(y)_{+}=(y+|y|) / 2$ requires no branching. But, the truncated powers are an ill-conditioned way of representing splines (ibid, pp.17-19, pp.104105), and the divided difference can not only require branching for near-coalescence but has additional conditioning problems. So, we propose to calculate $f_{\nu}(\mathrm{A} 4.1)$ in two other ways.

For the first: Suppose the left-most knot in $T=T(S, \nu)$ has multiplicity $l$ $\left(1 \leq l \leq n\right.$ since $S$ is nondegenerate); i.e., suppose $t_{0}=\cdots=t_{l-1}<t_{l}$. Extend the sequence $T$ to the left by adjoining $n+1-l$ additional knots equal to $t_{0}$, so that $t_{0}$ has multiplicity $n+1$ in the extension (actually, any suitably ordered $n+1-l$ extra knots would do in principle, but this choice insures that numerical conditioning is not made worse, $\mathrm{cf}$. de Boor [6]):

$$
t_{l-1-n}=\cdots=t_{0}=\cdots=t_{l-1}<t_{l} \leq \cdots \leq t_{n}
$$

Proposition A 4.1. For $-\sigma$ in $\left(t_{0}, t_{n}\right)$ and with $l(\leq n)$ the multiplicity of $t_{0}$ in $T(S, \nu)$, the cumulative distribution function (A4.1) is the sum of the $n-l+1$ 
$B$-splines of order $n+1$ (degree $n)$ associated with the knot sequence (A4.3):

$$
f_{\nu}(\sigma ; S)=\sum_{j=l-1-n}^{-1} B_{j, n+1}(-\sigma), \quad t_{0}<-\sigma<t_{n} .
$$

(Of course, $f_{\nu}$ is one for $-\sigma \leq t_{0}$ and zero for $-\sigma \geq t_{n}$.)

Proof. Although this follows, using $M_{T}=\sum_{j} \delta_{0 j}\left(n /\left(t_{n+j}-t_{j}\right)\right) B_{j, n}$, from de Boor's [5, pp. 150-151] discussion of the integration of a spline represented by its B-spline coefficient sequence, we verify it directly. Suppose $t_{n}$ had multiplicity $m$ in $T$. Extend the knot sequence (A4.3) to the right by adjoining $n+1-m$ more knots equal to $t_{n}$, so that also

$$
t_{n-m}<t_{n-m+1}=\cdots=t_{n}=\cdots=t_{2 n+1-m} .
$$

The coefficients of the B-splines $B_{i, n+1}$ which are relevant to the values of a spline $\sum_{i} d_{i} B_{i, n+1}$ on $\left(t_{0}, t_{n}\right)=\left(t_{l-1}, t_{n-m+1}\right)$ are

$$
\left(d_{l-n-1}, \ldots, d_{-1}, d_{0}, \ldots, d_{n-m}\right) ; \quad \text { i.e., }(1, \ldots, 1,0, \ldots, 0) \text { for (A4.4). }
$$

But, the coefficients of the $n$ th-order B-splines relevant to this spline's derivative $\sum c_{i} B_{i, n}$ on $\left(t_{0}, t_{n}\right)=\left(t_{l-1}, t_{n-m+1}\right)$ are $c_{l-n}, \ldots, c_{n-m}$. According to de Boor [3], [5, p. 138], the B-spline coefficients of the first derivative of an $(n+1)$ th-order spline $\sum d_{i} B_{i, n+1}$ are given by the first-order de Boor difference-quotient (as I call it in Swartz $\left[25\right.$, p.135]) sequence $\left(\Delta_{b}^{1} d\right)_{i}:=\left[\left(d_{i}-d_{i-1}\right) /\left(\left(t_{i+n}-t_{i}\right) / n\right)\right]$ of its coefficients; hence, here, by

$$
\left(c_{l-n}, \ldots c_{-1}, c_{0}, c_{1}, \ldots, c_{n-m}\right)=\left(0, \ldots, 0,-n /\left(t_{n}-t_{0}\right), 0, \ldots, 0\right)
$$

( $c_{0} \neq 0$ taking precedence in ambiguous cases). As we have already noted, this is the negative (as is required) of the coefficient sequence of $M_{T(S, \nu)}$ relevant to $\left(t_{0}, t_{n}\right)$.

It remains to show that (A4.4) is not off by an additive constant. But $t_{n}$ has multiplicity $m \leq n$-as the final knot not only in $T$, but also in the right-most B-spline $B_{-1, n+1}$ in (A4.4). Hence, the limit of each term there, as $-\sigma \uparrow t_{n}$, is zero.

Assuming de Boor's B-spline program package is available, (A4.3)-(A4.4) is easy to code. But that package can also produce local representation of the (up to) $n$ polynomial pieces which together comprise $M_{T(s, \nu)}=n B_{0, n} /\left(t_{n}-t_{0}\right)$, pieces which could then be integrated as appropriate to the computation of (A4.1) and the resulting code could be more efficient. Along these last lines, we supply (in the Supplement) a computer program which, for $n=3$ dimensions and given the nondecreasing quadruple $T(S, \nu)$, evaluates (A4.1) without recourse to de Boor's package. This code is based on the fact that the three columns in $\operatorname{diag}(1,2,1)$ times the final matrix-product in Swartz [25, p.146] are, when suitably interpreted, the coefficients of the polynomial pieces of $B_{0,3}$. Thus with $h_{+}:=t_{3}-t_{2}$ assumed positive, $h_{0}:=t_{2}-t_{1} \geq 0, \bar{t}_{23}:=\left(t_{2}+t_{3}\right) / 2, x:=\left(2 h_{0}+h_{+}\right) / h_{+}$, and $y:=1$, the matrix's first column constitutes the coefficients of $1, \eta, \eta^{2}$ for the quadratic $q(\eta)=B_{0,3}\left(\bar{t}_{23}+h_{+} \eta / 2\right),-1_{+} \leq \eta \leq 1_{-}$. The second and third columns yield, with all quantities suitably redefined, the quadratics on the middle and first intervals, respectively. 
Finally, consider the computation of the cubic spline which represents, for a given normal $\nu$, the cumulative distribution function for $\nu$-normal planes associated with the 3-cube of unit mass having vertices $c_{k}=k / 2, k:=( \pm 1, \pm 1, \pm 1)$. Given the general discussion above (and the computer code in particular), it suffices to recall the decomposition of the cube into five interiorly disjoint tetrahedra. Four of these tetrahedra, $S_{1}, \ldots, S_{4}$, will be congruent, "corner-cut-off" tetrahedra-each with basal area $1 / 2$, hence mass $1 / 6$. The fifth, centrally located tetrahedron $S_{5}$-with its consequent mass of $1 / 3$-is regular, i.e., equisided, and determines the particular decomposition. Its six (1-dimensional) edges are diagonals of the cube's six faces, with one diagonal per face-thus, choose a face and a diagonal on it, and the rest is determined.

On the other hand, Youngs [29] presents formulas for the five special cases he associates with this cumulative distribution function for a 3-cube.

Zemach [30] has derived the cumulative distribution function for $\nu$-normal planes of a 3-dimensional "hexahedron" - that generalization of a cube each of whose "faces" is the ruled surface spanning its four associated (and appropriately ordered) vertices.

Acknowledgments. The author is indebted to Burton Wendroff for asking how one should approximately reconstruct an interface from the volume fractions of computational hydrodynamics. He has had fruitful conversation, too, with $\mathrm{W}$. Beyer, P. de Boor, A. Brandt, R. Hersh, N. Johnson, J. Louck, T. Manteuffel, R. Mjolsness, M. Wing, and C. Zemach.

Theoretical Division, MS B284

University of California

Los Alamos National Laboratory

Los Alamos, New Mexico 87545

E-mail: bks@lanl.gov

1. W. A. BEYER \& B. SWARTz, Halfway Points (a report in preparation), Los Alamos National Laboratory, Los Alamos, NM, 1989.

2. C. DE BOOR, Subroutine Package for Calculating with B-splines, Report LA-4728-MS, Los Alamos Scientific Laboratory, 1971, 12 pp. (see also SIAM J. Numer. Anal., v. 14, 1977, pp. 441472).

3. C. DE BOOR, "On calculating with B-splines," J. Approx. Theory, v. 6, 1972, pp. 50-62.

4. C. DE BOOR, "Splines as linear combinations of B-splines," in Approximation Theory II (G. G. Lorentz, C. K. Chui \& L. L. Schumaker, eds.), Academic Press, New York, 1976, pp. 1-47.

5. C. DE BOor, A Practical Guide to Splines, Appl. Math. Sci. \#27, Springer-Verlag, New York, 1978.

6. C. DE BOOR, "The condition of the B-spline basis for polynomials," SIAM J. Numer. Anal., v. 25, 1988, pp. 148-152.

7. D. S. Carter, G. Pimbley \& G. M. Wing, On the Unique Solution for the Density Function in Phermex, (Declassified) Memo T-5-2023, Los Alamos Scientific Laboratory, Los Alamos, NM, 1957, $7 \mathrm{pp}$.

8. A. J. Chorin, "Curvature and solidification," J. Comput. Phys., v. 57, 1985, pp. 472-490.

9. M. G. Cox, "The numerical evaluation of B-splines," J. Inst. Math. Appl., v. 10, 1972, pp. 134-149.

10. H. B. CurRY \& I. J. SChoenberg, "On Polya frequency functions. IV: The fundamental spline functions and their limits," J. Analyse Math., v. 17, 1966, pp. 71-107.

11. R. B. DEBAR, Fundamentals of the KRAKEN Code, Report UCID-17366, Lawrence Livermore Laboratory, Livermore, CA, 1974, 17pp. 
12. G. DE CECCO, "Il teorema del sandwich al prosciutto," Archimede, v. 37, 1985, pp. 98-106. (Italian)

13. V. FABER \& G. M. WING, The Abel Integral Equation, Report LA-11016-MS, Los Alamos National Laboratory, Los Alamos, NM, 1987, 48pp.

14. C. FENSKE, Math. Rev., 87h:54078, 1987.

15. G. H. GolUB \& C. F. VAN LOAN, Matrix Computations, Johns Hopkins Univ. Press, Baltimore, MD, 1983.

16. K. HÖllig, "Multivariate splines," in Approximation Theory (C. de Boor, ed.), AMS Short Course Lecture Notes \#36, Amer. Math. Soc., Providence, R.I., 1986, pp. 103-127.

17. B. R. HUN T (editor), "Special image processing issue," Proc. IEEE, v. 69, 1981, pp. $499-655$.

18. A. HUXLEY, An Illustrated History of Gardening, Paddington Press (Grosset and Dunlap), New York, 1978.

19. J. M. HYMAN, "Numerical methods for tracking interfaces," in Fronts, Interfaces and Patterns

(A. R. Bishop, L. J. Campbell \& P. J. Channell, eds.), Elsevier, New York, 1984, pp. 396-407.

20. H.-O. Kreiss, T. A. MAnteuffel, B. SWARtZ, B. WendrofF \& A. B. White, "Supraconvergent schemes on irregular grids," Math. Comp., v. 47, 1986, pp. 537-554.

21. R. C. MJOlsness \& B. SWARTz, "Some plane curvature approximatons," Math. Comp., v. 49, 1987, pp. 215-230.

22. B. D. Nichols \& C. W. HIRT, Methods for Calculating Multi-Dimensional, Transient Free Surface Flows Past Bodies, Proc. First Internat. Conf. Numer. Ship Hydrodynamics, Gaithersburg, MD, 1975.

23. W. F. NOH \& P. WOOdWARD, "SLIC (Simple Line Interface Calculation)," in Proc. Fifth Internat. Conf. on Numer. Methods in Fluid Dynamics, Lecture Notes in Physics (A. I. van der Vooren \& P. J. Zandbergen, eds.), Springer-Verlag, New York, 1976, pp. 330-340.

24. B. SWARTZ, The Second-Order Sharpening of Blurred Smooth Borders, Report LA-UR-87-2933, Los Alamos National Laboratory, 1987, 35pp.

25. B. SWARTZ, "Conditioning collocation," SIAM J. Numer. Anal., v. 25, 1988, pp. 124-147.

26. G. M. WING, A Primer on Integral Equations of the First Kind, Report LA-UR-84-1234, 1984, 98pp.

27. D. L. YOUNGS, private communication, 1978.

28. D. L. YoungS, "Time-dependent multi-material flow with large fluid distortion," Numerical Methods for Fluid Dynamics (K. W. Morton \& M. J. Baines, eds.), Academic Press, New York, 1982, pp. 274-285.

29. D. L. Youngs, An Interface Tracking Method for a $3 D$ Eulerian Hydrodynamics Code, Atomic Weapons Research Establishment Report AWRE-44-92-35, Aldermaston, Berks., 1987, 47pp.

30. C. ZEMACH, T-Division, Los Alamos National Laboratory; private communication, 1988. 


\title{
Supplement to The Second-Order Sharpening of Blurred Smooth Borders
}

\author{
By Blair Swartz
}

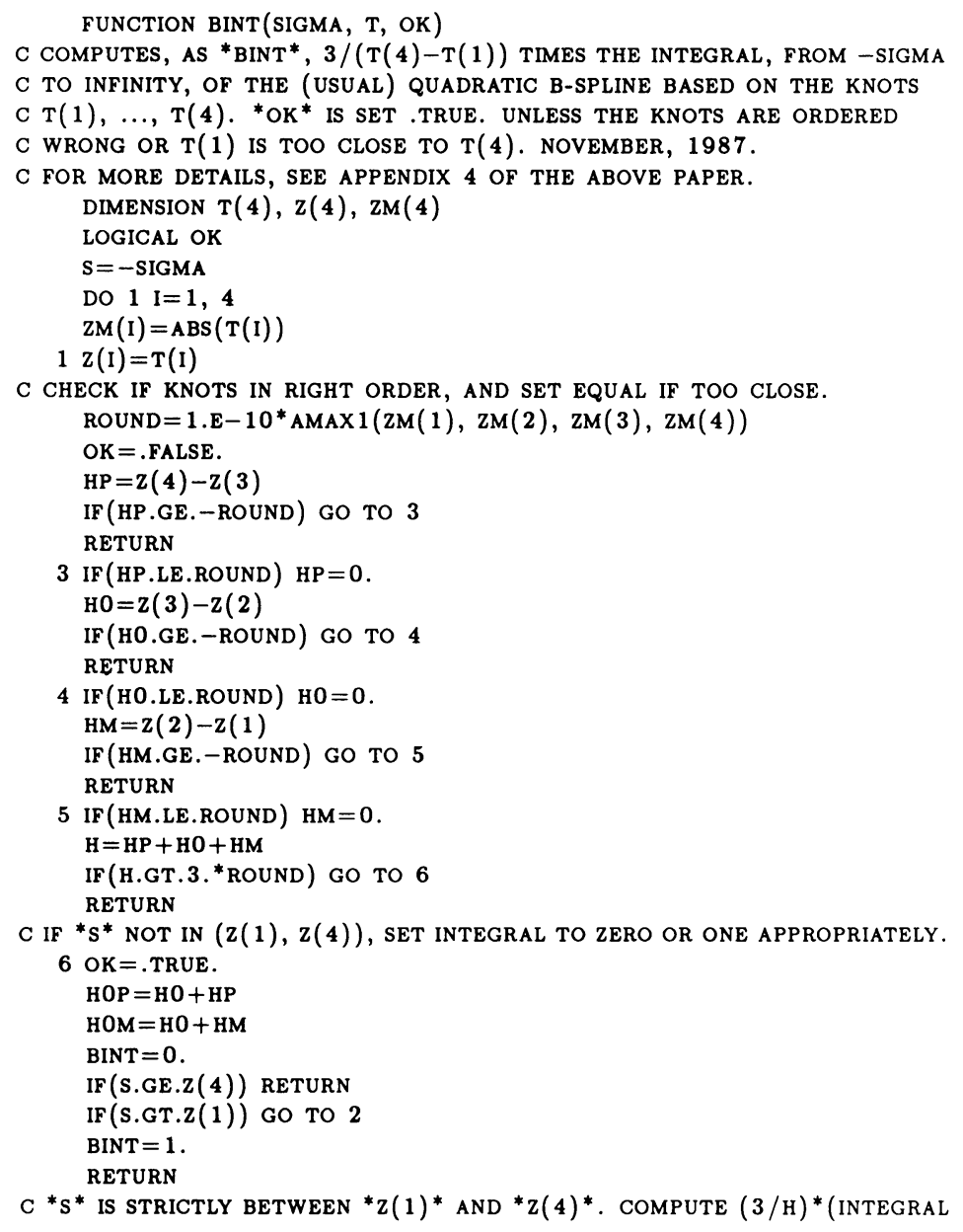




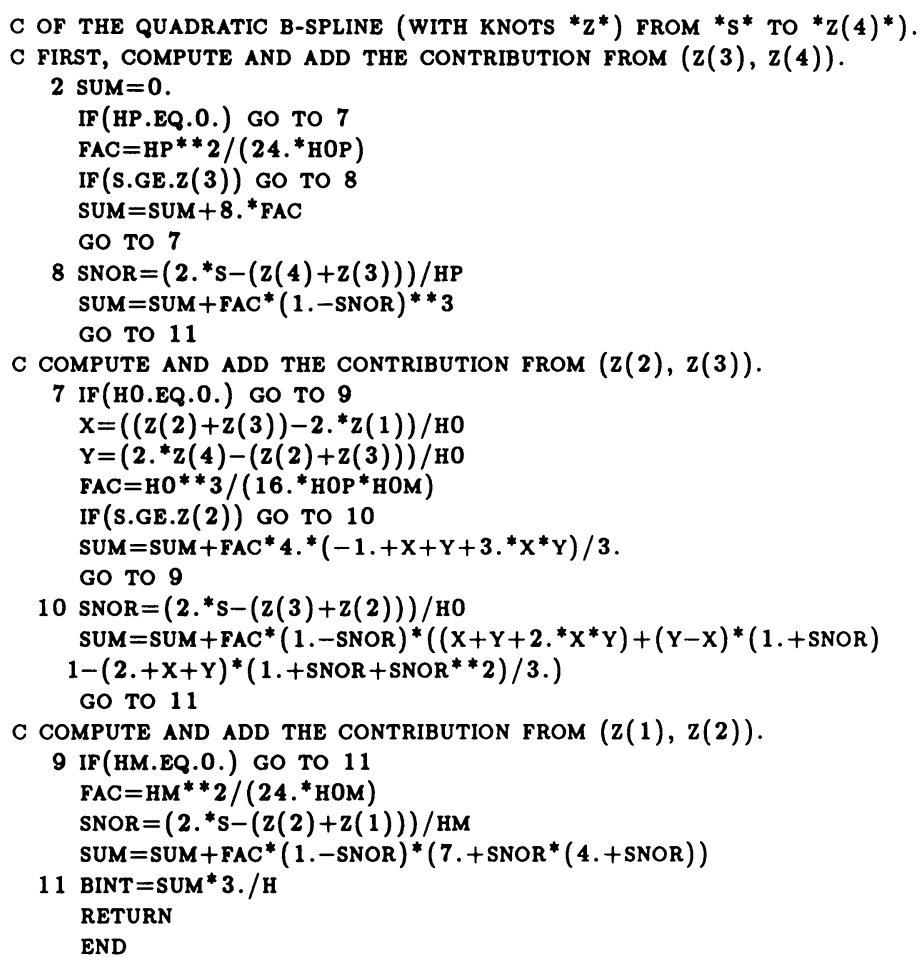

